\title{
TWO CHARACTERISTIC NUMBERS FOR SMOOTH PLANE CURVES OF ANY DEGREE
}

\author{
PAOLO ALUFFI
}

\begin{abstract}
We use a sequence of blow-ups over the projective space parametrizing plane curves of degree $d$ to obtain some enumerative results concerning smooth plane curves of arbitrary degree. For $d=4$, this gives a first modern verification of results of $\mathbf{H}$. G. Zeuthen.
\end{abstract}

\section{INTRODUCTION}

The $k$ th 'characteristic number' of the $\frac{d(d+3)}{2}$-dimensional family of smooth plane curves of degree $d$, denoted $N_{d}(k)$ in the following, is the number of such curves which are tangent to $k$ lines and contain $\frac{d(d+3)}{2}-k$ points in general position in the plane. Elementary considerations and Bézout's theorem (see $\S 1$ below) show that $N_{d}(k)=(2 d-2)^{k}$ for $k<2 d-1$.

In this paper we compute the next two cases as a closed form in terms of the degree $d$. Our result is

$$
\begin{gathered}
N_{d}(2 d-1)=(2 d-2)^{2 d-1}-2^{d-3} d(d-1)\left(d^{2}-d+2\right), \\
N_{d}(2 d)=(2 d-2)^{2 d}-2^{d-4} d(d-1)\left(8 d^{4}-21 d^{3}+19 d^{2}-20 d+32\right) .
\end{gathered}
$$

Also, for $d=4$ we obtain the next characteristic number $N_{4}(9)=9,840,040$.

The characteristic numbers of a family are its basic enumerative information; the problem of computing them for families of plane curves has received quite some attention in the recent past. For the family of smooth plane curves of degree $d$, the modern literature lists the numbers $N_{2}(k), N_{3}(k)$ for smooth conics and cubics $[\mathrm{F}, \mathrm{A}, \mathrm{KS}]$; for $d=4$, the numbers $N_{4}(7)=279,600$, $N_{4}(8)=1,668,096$ and $N_{4}(9)=9,840,040$ computed here verify classic results of H. G. Zeuthen's [Z] (in which-among many others-all the characteristic numbers $N_{4}(k)$ for smooth plane quartics are obtained). For degree $\geq 5$, the results of this paper seem to be new (we know of recent work of Leendert van Gastel on this problem, from a different viewpoint).

Our approach is in the spirit of the computation of the characteristic numbers for smooth plane cubics in $[\mathrm{A}]$. Let $\mathbb{P}^{N}$ be the projective space parametrizing plane curves of degree $d$. Call 'point-condition' the hyperplane in $\mathbb{P}^{N}$ formed by the curves $C \in \mathbb{P}^{N}$ which contain a given point, and 'line-condition' the hypersurface (of degree $2 d-2$ ) consisting of the curves $C \in \mathbb{P}^{N}$ which are

Received by the editors July 21, 1989 and, in revised form, March 8, 1990.

1980 Mathematics Subject Classification (1985 Revision). Primary 14N10; Secondary 14C17.

Key words and phrases. Enumerative geometry, plane curves, blow-up. 
tangent to a given line. The intersection of all line-conditions is supported on the set $S \subset \mathbb{P}^{N}$ consisting of all curves $C \in \mathbb{P}^{N}$ containing a multiple component.

Let now $\widetilde{V}$ be a smooth variety mapping birationally onto $\mathbb{P}^{N}$, and denote by $\widetilde{P}$ and $\widetilde{L}$ the classes of the proper transforms of the general point- and line-conditions. In [A, Corollary I], we observed that if the intersection of the proper transforms of all line-conditions is empty, then $N_{d}(k)=\widetilde{P}^{N-k} \cdot \widetilde{L}^{k}$. We call such a $\widetilde{V}$ a smooth variety of 'complete plane curves of degree $d$.' The computation of the numbers for smooth plane cubics in [A] is achieved by constructing a variety of complete cubics by a sequence of five blow-ups along smooth centers over $\mathbb{P}^{9}$.

The point is to construct $\widetilde{V}$ while maintaining control of its Chow ring; this can be accomplished e.g. by blowing-up along smooth centers. In this sense, constructing a 'nice' variety of complete curves of degree $d$ for $d \geq 4$ seems a formidable task. However, for a specific $k$, it suffices to construct the variety over an open set of $\mathbb{P}^{N}$ containing the intersection of $k$ line-conditions and $N-k$ point-conditions chosen generally; for example, $\mathbb{P}^{N}$ itself can be used if $k \leq 2 d-2$. For the results in this note (i.e., $k \leq 2 d$ ), we will consider an open set basically big enough to contain the smooth part of the set $B \subset \mathbb{P}^{N}$ consisting of curves decomposing into a 'double line' and a curve of degree $d-2$. For all $d$, we will use two blow-ups to construct a variety $\widetilde{V}$ satisfying our requirements for $k \leq 2 d-1$ (§3). For $k=2 d$, a third blow-up (along a nonreduced center for $d>4$ ) would be necessary to construct the variety over the locus consisting of curves decomposing into a curve of degree $d-2$ and a double line tangent to the curve. However, this last step amounts to the computation of the contribution of isolated points to the intersection number of $N$ divisors in $\widetilde{V}$, and it seems easier to evaluate this contribution directly $(\S 4)$.

For $d=4$ it is easy to analyze the third blow-up and construct the variety over the set of quartics consisting of a smooth conic and a double line tangent to it. As a bonus we get a third characteristic number in this case, after evaluating directly the contribution due to a double conic and to quartics consisting of a triple of lines, one of which double, meeting at a point $(\S 5)$.

The two blow-ups we give in $\S 3$ generalize to arbitrary degree the last two blow-ups considered in [A] for $d=3$. As in [A], after constructing the variety $\widetilde{V}$ as a sequence of blow-ups, we compute $\widetilde{P}^{N-k} \cdot \widetilde{L}^{k}, k=2 d-1,2 d$, by using an intersection formula involving some information about the normal bundles of the centers of the blow-ups $(\S 4)$.

A technical difficulty in this approach to the computation is the determination of the intersection of all proper transforms of line-conditions at each new blowup. Heuristically speaking, more blow-ups are needed over points at which the scheme-intersection of all line-conditions is richer in structure; an essential step in our computation is the estimation of this factor. Let $\mathscr{S}$ be a subscheme of a smooth variety, and $p$ a simple point of the support $S$ of $\mathscr{S}$. We define the 'thickness' of $\mathscr{S}$ at $p, \operatorname{th}_{p}(\mathscr{S})$ to be the maximum length of the intersection of $\mathscr{S}$ with a curve germ centered at $p$ and transversal to $S$. For $S \subset \mathbb{P}^{N}$ denoting (as above) the set of nonreduced curves and $\mathscr{S}$ the scheme-theoretic intersection of all line-conditions supported on $S$, we compute $\operatorname{th}_{p}(\mathscr{S})$ in $\S 2$ 
(Lemma II) for $p \in \mathbb{P}^{N}$ corresponding to certain plane curves decomposing into a double line $\mu$ and a curve $C$ of degree $d-2$. We get $\operatorname{th}_{p}(\mathscr{S})=2$ if $\mu$ is not tangent to $C$, and $\operatorname{th}_{p}(\mathscr{S})=3$ if $\mu$ is tangent to $C$ at a single smooth point of $C$. This information is used crucially to show that two blow-ups suffice for $k=2 d-1$, and to gather information necessary to treat the case $k=2 d$.

It should be pointed out that in fact the case $k=2 d-1$ amounts to the evaluation of the contribution to the intersection multiplicity of $N$ divisors in $\mathbb{P}^{N}$ due to isolated points of intersection (corresponding to singular curves). Given the geometry of the situation (the tangent spaces to the conditions intersect in a $d$-dimensional space), the minimum that the contribution of each point 'could be' is $2^{d}$ (see the remark in $\S 4$ ); our result for $k=2 d-1$ shows that this is precisely the case. For $k=2 d$, we are computing the total contribution of a union of curves to the intersection number of $N$ divisors in $\mathbb{P}^{N}$. It would be interesting to interpret this result similarly, in terms of simple geometry considerations, as a 'minimal allowed' contribution.

I would like to thank Alberto Collino, William Fulton, Joe Harris, and Sheldon Katz for several inspiring conversations.

\section{BASIC FACTS AND NOTATIONS}

Let $\mathbb{V}$ be a three-dimensional complex vector space, $\mathbb{P}^{2}=\mathbb{P}(\mathbb{V})$, and $\mathbb{P}^{N}=$ $\mathbb{P}\left(\operatorname{Sym}^{d \check{V}}\right)$ the projective space of dimension $N=\frac{d(d+3)}{2}$ parametrizing plane curves of degree $d$. In the following it will be convenient to assume $d \geq 4$; however, the main results hold as stated for conics and cubics as well.

If $p$ is a point in the plane, the curves that contain $p$ determine a hyperplane $P_{p}$ in $\mathbb{P}^{N}$ : a 'point-condition' in $\mathbb{P}^{N}$. Similarly, the curves tangent to a given line $l$ form a hypersurface $L_{l}$ of degree $2 d-2$ : a 'line-condition' in $\mathbb{P}^{N}$. If $p_{1}, \ldots, p_{N-k}$ and $l_{1}, \ldots, l_{k}$ are general points and lines, we are interested in the number $N_{d}(k)$ of smooth curves containing $p_{1}, \ldots, p_{N-k}$ and tangent to $l_{1}, \ldots, l_{k}$, i.e., the number of points in the intersection $P_{p_{1}} \cap \cdots \cap P_{p_{N-k}} \cap L_{l_{1}} \cap$ $\cdots \cap L_{l_{k}}$ that correspond to smooth curves.

Suppose now that $\widetilde{V}$ is a variety mapping birationally to $\mathbb{P}^{N}$, such that the intersection of the proper transforms in $\widetilde{V}$ of all line-conditions is empty. We call any such variety a 'variety of complete curves of degree $d$ ' (such varieties exist; for example, blow-up $\mathbb{P}^{N}$ along the scheme-theoretic intersection of the line-conditions). The proper transforms of the general point-condition $P$ and line-condition $L$ determine divisor classes $\widetilde{P}, \widetilde{L}$ in $\widetilde{V}$; we observed in [A, Corollary I], that if $\widetilde{V}$ is a variety of complete curves, then $N_{d}(k)=\widetilde{P}^{N-k} \cdot \widetilde{L}^{k}$ for all $k$. We will use this fact in a more specific formulation.

For any variety $\widetilde{V}$ mapping birationally to $\mathbb{P}^{N}$, call 'point-conditions' and 'line-conditions' in $\widetilde{V}$ the proper transforms of the point- and line-conditions in $\mathbb{P}^{N}$. Denote as above by $\widetilde{P}$ and $\widetilde{L}$ the divisor classes of the general pointand line-condition in $\widetilde{V}$.

Lemma I. Suppose $\widetilde{V} \stackrel{\pi}{\rightarrow} \mathbb{P}^{N}$ is a birational morphism such that, for general point-conditions $P_{1}, \ldots, P_{N-k}$ in $\mathbb{P}^{N}$, the intersection of all line-conditions in $\widetilde{V}$ is disjoint from $\pi^{-1}\left(P_{1} \cap \cdots \cap P_{N-k}\right)$. Then $N_{d}(k)=\widetilde{P}^{N-k} \cdot \widetilde{L}^{k}$.

Proof. Let $L_{1}, \ldots, L_{k}$ be general line-conditions in $\mathbb{P}^{N}$. If $\widetilde{P}_{i}, \widetilde{L}_{j}$ are the proper transforms of $P_{i}, L_{j}$ in $\widetilde{V}$, the hypotheses guarantee that $\widetilde{V}$ is iso- 
morphic to a variety of complete curves of degree $d$ in a neighborhood of $\widetilde{P}_{1} \cap \cdots \cap \widetilde{P}_{N-k} \cap \widetilde{L}_{1} \cap \cdots \cap \widetilde{L}_{k}$. The claim follows then from the fact that $\widetilde{P}^{N-k} \cdot \widetilde{L}^{k}$ is a sum of local contributions.

The intersection of all line-conditions on $\mathbb{P}^{N}$ is supported on the set $S$ consisting of curves with multiple components. The structure of $S$ is in general very complicated; however, for our purposes the relevant observation is quite simple:

Lemma 1.1. The highest dimensional component in $S$ is the set $B$ formed by curves containing a double line. $B$ has codimension $2 d-1$ in $\mathbb{P}^{N}$; the other components of $S$ and the singular locus of $B$ have codimension $>2 d$.

Proof. $B$ is the image of a map

$$
\begin{gathered}
\mathbb{P}^{(d-2)(d+1) / 2} \times \check{\mathbb{P}}^{2} \stackrel{i}{\rightarrow} \mathbb{P}^{d(d+3) / 2} \\
(C, \mu) \mapsto C \mu^{2},
\end{gathered}
$$

where $\mathbb{P}^{(d-2)(d+1) / 2}=\mathbb{P}\left(\operatorname{Sym}^{d-2} \check{\mathbb{V}}\right)$ parametrizes plane curves of degree $d-2$, and $\check{\mathbb{P}}^{2}$ parametrizes lines. One verifies easily that $i$ is an embedding at points $(C, \mu)$ with $C$ reduced and not containing $\mu$ ( $d i$ is injective if $C$ does not contain $\mu$ ). The statement follows then from simple dimension computations.

We will denote by $C \mu^{2}$ a point of $B$ decomposing into the degree- $(d-2)$ curve $C$ and the double line supported on the line $\mu$. As observed in the proof, $B$ is smooth at $C \mu^{2}$ e.g. if $C$ is reduced and intersects $\mu$ properly.

Lemma I gives immediately

Proposition 1.2. For $k<2 d-1, N_{d}(k)=(2 d-2)^{k}$.

Proof. Indeed, for $k<2 d-1$ and $N-k$ general point-conditions $P_{1}, \ldots$, $P_{N-k}$ in $\mathbb{P}^{N}, P_{1} \cap \cdots \cap P_{N-k} \cap S=\varnothing$ (this follows from Lemma 1.1 and e.g. from Remark $1, \S 1$ in [A]). We can then apply Lemma I to $\widetilde{V}=\mathbb{P}^{N}$ and $\pi=$ identity, and compute $\widetilde{P}^{N-k} \cdot \widetilde{L}^{k}$ using Bézout's theorem.

For $k \geq 2 d-1$, the intersection of $N-k$ point-conditions is never disjoint from $S$. In $\S 3$ we will construct a $\widetilde{V}$ fitting the hypotheses of Lemma I for $k=2 d-1$, by two successive blow-ups over $\mathbb{P}^{N}$.

Lemma 1.3. (1) For $k=2 d-1$ and $P_{1}, \ldots, P_{N-k}$ general point-conditions in $\mathbb{P}^{N}, P_{1} \cap \cdots \cap P_{N-k} \cap S$ consists of $\frac{d(d-1)\left(d^{2}-d+2\right)}{8}$ points $C \mu^{2} \in B$, with $C$ smooth and $\mu$ transversal to $C$.

(2) For $k=2 d$ and $P_{1}, \ldots, P_{N-k}$ general point-conditions in $\mathbb{P}^{N}, P_{1} \cap \cdots \cap$ $P_{N-k} \cap S$ consists of 1-dimensional subsets of $B$. All $C \mu^{2} \in P_{1} \cap \cdots \cap P_{N-k} \cap S$ have $C$ reduced, and $\mu$ intersecting $C$ properly, at smooth points of $C$. For finitely many such $C \mu^{2}, \mu$ will be simply tangent (at a single point) to $C$.

In particular, for $k=2 d-1$ or $2 d$ and $P_{1}, \ldots, P_{N-k}$ general pointconditions in $\mathbb{P}^{N}, P_{1} \cap \cdots \cap P_{N-k} \cap S$ is entirely contained in the smooth part of $B$.

Proof. Both (1) and (2) follow again easily from [A, Remark 1, §1], and dimension counts. The point is that one can choose $N-k$ point-conditions such that 
$P_{1} \cap \cdots \cap P_{N-k}$ is disjoint from given subsets of $\mathbb{P}^{N}$ of codimension $>k$. For example, as observed in Lemma 1.1 all components of $S$ other than $B$ and the singular locus of $B$ have codimension $>2 d$; therefore, the intersection of $N-2 d$ or more general point-conditions will miss these loci. As for the number $\frac{d(d-1)\left(d^{2}-d+2\right)}{8}$, this is the number $\left(\begin{array}{c}\left(\begin{array}{c}d \\ 2\end{array}\right)+1 \\ 2\end{array}\right)$ of lines containing two out of $\frac{d(d+3)}{2}-2 d+1=\left(\begin{array}{l}d \\ 2\end{array}\right)+1$ general points.

In view of Lemma $1.3(1)$, to apply Lemma I for $k=2 d-1$ we need to produce a variety $\widetilde{V}$ and a birational morphism $\pi: \widetilde{V} \rightarrow \mathbb{P}^{N}$, such that the intersection of all line-conditions in $\widetilde{V}$ is disjoint from each fiber $\pi^{-1}\left(C \mu^{2}\right)$ with $\mu$ a line and $C$ a reduced curve of degree $d-2$ intersecting $\mu$ transversally. As we will see, two blow-ups over $\mathbb{P}^{N}$ will produce a variety $\widetilde{V}$ satisfying this requirement. $\widetilde{V}$ will not suffice for $k=2 d$; as we shall see, the intersection of all line-conditions meets (at one point) the fiber over $C \mu^{2}$ when $\mu$ is tangent to $C$. However, the contribution given by this residual intersection can be computed directly.

\section{THICKNESS}

Let $\mathscr{S}$ be a subscheme of a smooth variety $V$, and $p$ a simple point of the support $S$ of $\mathscr{S}$. We define the 'thickness' of $\mathscr{S}$ at $p, \operatorname{th}_{p}(\mathscr{S})$, to be the maximum length of the intersection of $\mathscr{S}$ with a curve germ centered at $p$ and transversal to $S$. In this section we compute the 'thickness' $\operatorname{th}_{p}(\mathscr{S})$ of the scheme-theoretic intersection $\mathscr{S}$ of all line-conditions in $\mathbb{P}^{N}$ at points $p$ of $B$. Our basic observation is: suppose $\mathscr{S}$ is cut out by smooth hypersurfaces $H_{1}, \ldots, H_{m}$, let $\widetilde{V} \stackrel{\pi}{\rightarrow} V$ be the blow-up of $V$ along $S$, and denote by $\widetilde{H}_{i}$ the proper transform of $H_{i}$; also, denote by $\widetilde{\mathscr{S}}$ the scheme cut out by $\widetilde{H}_{1}, \ldots, \widetilde{H}_{m}$ in $\widetilde{V}$. Then, for $p \in S, \widetilde{\mathscr{S}} \cap \pi^{-1}(p) \neq \varnothing \Leftrightarrow \operatorname{th}_{p}(\mathscr{S}) \geq 2:$ indeed, $\operatorname{th}_{p}(\mathscr{S}) \geq 2$ precisely when there is a direction normal to $S$ and tangent to all the $H_{i}$. Also, if $\tilde{p} \in \widetilde{\mathscr{S}} \cap \pi^{-1}(p)$, then in good hypotheses $\operatorname{th}_{\tilde{p}}(\widetilde{\mathscr{S}})<\operatorname{th}_{p}(\mathscr{S}$ ) (in our applications these facts will follow directly from the definition). The result of our computation will be needed at several places in $\S \S 3$ and 4; for the moment, the hasty reader may want to assume Lemma II as stated below and skip the rest of this section.

We keep the notation of $\S 1: \mathbb{P}^{N}$ is the projective space parametrizing degree$d$ plane curves, and we call the 'line-condition' corresponding to a line $l$ the hypersurface of $\mathbb{P}^{N}$ consisting of all curves tangent to $l$. The intersection of all line-conditions in $\mathbb{P}^{N}$ is supported on the set formed by curves containing a multiple component: $B$ denotes the subvariety of $\mathbb{P}^{N}$ consisting of curves $C \mu^{2}$ decomposing in a degree- $(d-2)$ curve and a 'double line.'

Let $C \mu^{2} \in B \subset \mathbb{P}^{N}$, with $C$ a reduced curve of degree $d-2$ and $\mu$ a line intersecting $C$ at finitely many smooth points (we noticed in $\S 1$ that $B$ is nonsingular at such a $\left.C \mu^{2}\right)$. The intersection of all line-conditions is a scheme $\mathscr{S}$, one of whose components is supported on $B$. Denote by $\operatorname{th}_{C \mu^{2}}(\mathscr{S})$ (the 'thickness' of $\mathscr{S}$ at $C \mu^{2}$ ) the maximum length of the intersection with $\mathscr{S}$ at $C \mu^{2}$ of the germ of a smooth curve centered at $C \mu^{2}$ and transversal to $B$. Also, if $X$ is a plane curve not containing $\mu$, denote by $X_{\mu}$ the divisor cut by $X$ on $\mu$. We are going to show: 
Lemma II. (1) If $\mu$ is transversal to $C$, then $\operatorname{th}_{C \mu^{2}}(\mathscr{S})=2$.

(2) If $\mu$ is simply tangent to $C$ at precisely one smooth point, then $\operatorname{th}_{C \mu^{2}}(\mathscr{S})$ $=3$.

(3) In case (2), the thickness is 3 only along directions $C \mu^{2}+t K \mu$ with $K$ a degree- $(d-1)$ curve such that $K_{\mu}^{2} \geq C_{\mu}$.

(1), (2), and (3) deal with curve germs and certain hypersurfaces in the $\mathbb{P}^{N}$ parametrizing degree- $d$ curves in $\mathbb{P}^{2}$; we first analyze an analogous situation in the $\mathbb{P}^{d}$ parametrizing degree- $d$ effective divisors in $\mathbb{P}^{1}$. In this $\mathbb{P}^{d}$ we have the discriminant hypersurface $\Delta_{d}$ : let $\alpha, \beta$ be homogeneous coordinates on $\mathbb{P}^{1}$, so that points in $\mathbb{P}^{d}$ are zeros of polynomials $c_{0} \alpha^{d}+c_{1} \alpha^{d-1} \beta+\cdots+c_{d} \beta^{d}$; then $\Delta_{d}$ is given by the vanishing of the discriminant $\Delta_{d}\left(c_{0}, c_{1}, \ldots, c_{d}\right)$ of such polynomials.

Let $\gamma$ be a curve germ centered at a general point $D$ in $\Delta_{d}$; up to linear transformations, we can assume $D$ is double (only) at $(0: 1)$, and write

$$
\gamma(t)=c_{0}(t) \alpha^{d}+c_{1}(t) \alpha^{d-1} \beta+\cdots+c_{d}(t) \beta^{d},
$$

with $c_{0}(0)=c_{1}(0)=0, c_{2}(0) \neq 0$. We are interested in conditions on the $c_{i}(t)$ 's related to the order of contact $\left(\Delta_{d}, \gamma\right)_{0}$ of $\gamma$ and $\Delta_{d}$ at $t=0$.

Claim. (i) $\left(\Delta_{d}, \gamma\right)_{0} \geq 2 \Leftrightarrow c_{0}^{\prime}(0)=0$;

(ii) $\left(\Delta_{d}, \gamma\right)_{0} \geq 3 \Leftrightarrow c_{0}^{\prime}(0)=0$ and $c_{1}^{\prime}(0)^{2}=2 c_{2}(0) c_{0}^{\prime \prime}(0)$;

(iii) $\left(\Delta_{d}, \gamma\right)_{0} \geq 4 \Leftrightarrow c_{0}^{\prime}(0)=0, c_{1}^{\prime}(0)^{2}=2 c_{2}(0) c_{0}^{\prime \prime}(0)$, and $2 c_{2}(0)^{2} c_{0}^{\prime \prime \prime}(0)-$ $3 c_{2}(0) c_{1}^{\prime}(0) c_{1}^{\prime \prime}(0)+6 c_{2}(0) c_{2}^{\prime}(0) c_{0}^{\prime \prime}(0)-3 c_{3}(0) c_{1}^{\prime}(0) c_{0}^{\prime \prime}(0)=0$.

Proof. The discriminant hypersurface is the projection of the codimension-2 subvariety of $\mathbb{P}^{d} \times \mathbb{P}^{1}$ defined by

$$
\left\{\begin{array}{c}
d c_{0} \alpha^{d-1}+(d-1) c_{1} \alpha^{d-2} \beta+\cdots+c_{d-1} \beta^{d-1}=0, \\
c_{1} \alpha^{d-1}+2 c_{2} \alpha^{d-2} \beta+\cdots+d c_{d} \beta^{d-1}=0 .
\end{array}\right.
$$

By the projection formula, $\left(\Delta_{d}, \gamma\right)_{0}$ is the intersection multiplicity of this variety with the germ of surface $(\gamma(t), s)$ in $\mathbb{P}^{d} \times \mathbb{P}^{1}$. In other words, $\left(\Delta_{d}, \gamma\right)_{0}$ is the intersection multiplicity at the origin of the curves

$$
\begin{gathered}
d c_{0}(t)+(d-1) c_{1}(t) s+\cdots+c_{d-1}(t) s^{d-1}=0, \\
c_{1}(t)+2 c_{2}(t) s+\cdots+d c_{d}(t) s^{d-1}=0 .
\end{gathered}
$$

in the $(s, t)$-plane.

Now observe that if $d>4$, then the term $c_{d}(t) s^{d-1}$ vanishes to order at least 4 at the origin: therefore this term is irrelevant to whether $\left(\Delta_{d}, \gamma\right)_{0} \leq 4$. Hence, if $d>4$ we may assume $c_{d}(t)=0 \quad \forall t$, i.e., we may assume that all divisors $\gamma(t)$ in $\mathbb{P}^{1}$ contain the point at infinity $(1: 0)$. Also, since $\gamma(0)$ was general we may assume $c_{d-1}(0) \neq 0$.

Next, observe $\Delta_{d}\left(c_{0}, \ldots, c_{d-1}, 0\right)=c_{d-1}^{2} \Delta_{d-1}\left(c_{0}, \ldots, c_{d-1}\right)$; therefore, if $d>4$ then the conditions for $d$ are the same as the conditions for $d-1$. I.e., in determining these conditions we may assume $d=4$, and a direct computation gives (i), (ii), and (iii).

Now consider a curve $\gamma(t)$ in $\mathbb{P}^{N}$ such that $\gamma(0)=C \mu^{2} \in B$ ( $C$ reduced, $\mu$ intersecting $C$ at finitely many smooth points), and $\gamma(0)$ is transversal to $B$ 
at 0 . We write

$$
\gamma(t)=P+Q t+R t^{2}+S t^{3}+\cdots,
$$

where $P, Q, R, \ldots \in \mathbb{P}^{N}$. Choosing homogeneous coordinates $\left(x_{0}: x_{1}: x_{2}\right)$ in $\mathbb{P}^{2}$, we will write e.g.

$$
P=P\left(x_{0}: x_{1}: x_{2}\right)=P_{d}\left(x_{1}: x_{2}\right)+P_{d-1}\left(x_{1}: x_{2}\right) x_{0}+P_{d-2}\left(x_{1}: x_{2}\right) x_{0}^{2}+\cdots,
$$

with $P_{i}\left(x_{1}: x_{2}\right)$ homogeneous polynomials of degree $i$ in $x_{1}, x_{2}$. In this notation,

$$
\begin{aligned}
\gamma(t) & =\left(P_{d}\left(x_{1}: x_{2}\right)+P_{d-1}\left(x_{1}: x_{2}\right) x_{0}+P_{d-2}\left(x_{1}: x_{2}\right) x_{0}^{2}+\cdots\right) \\
& +\left(Q_{d}\left(x_{1}: x_{2}\right)+Q_{d-1}\left(x_{1}: x_{2}\right) x_{0}+Q_{d-2}\left(x_{1}: x_{2}\right) x_{0}^{2}+\cdots\right) t \\
& +\left(R_{d}\left(x_{1}: x_{2}\right)+R_{d-1}\left(x_{1}: x_{2}\right) x_{0}+R_{d-2}\left(x_{1}: x_{2}\right) x_{0}^{2}+\cdots\right) t^{2}+\cdots .
\end{aligned}
$$

Assuming $\gamma(0)=C \mu^{2}$ imposes $P_{d}=P_{d-1}=0, P_{d-2} \neq 0$.

Let $l$ be a general line. Up to a linear transformation of the plane, we can assume $l$ has equation $l_{2} x_{1}-l_{1} x_{2}=0$, i.e., it contains the point $(1: 0: 0)$. Then $l$ is parametrized by $(\alpha: \beta)$ via $x_{0}=\beta, x_{1}=l_{1} \alpha, x_{2}=l_{2} \alpha$; for any $t$ the degree- $d$ curve $\gamma(t) \in \mathbb{P}^{N}$ cuts on $l$ the degree- $d$ divisor

$$
\begin{aligned}
\gamma_{l}(t)= & \left(P_{d-2}\left(l_{1}: l_{2}\right) \alpha^{d-2} \beta^{2}+\cdots\right) \\
& +\left(Q_{d}\left(l_{1}: l_{2}\right) \alpha^{d}+Q_{d-1}\left(l_{1}: l_{2}\right) \alpha^{d-1} \beta+Q_{d-2}\left(l_{1}: l_{2}\right) \alpha^{d-2} \beta^{2}+\cdots\right) t \\
& +\left(R_{d}\left(l_{1}: l_{2}\right) \alpha^{d}+R_{d-1}\left(l_{1}: l_{2}\right) \alpha^{d-1} \beta+R_{d-2}\left(l_{1}: l_{2}\right) \alpha^{d-2} \beta^{2}+\cdots\right) t^{2}+\cdots .
\end{aligned}
$$

Applying part (i) of the claim gives: $\gamma$ is tangent to the line-condition corresponding to $l$ at $C \mu^{2}$ if and only if $Q_{d}\left(l_{1}: l_{2}\right)=0$.

Therefore, $\gamma$ is tangent to all line-conditions if and only if $Q_{d}\left(l_{1}: l_{2}\right)=0$ for all $l_{1}, l_{2}$, i.e., if and only if $Q_{d}=0$. Assume this is the case, so that

$$
\gamma(t)=\begin{gathered}
\left(P_{d-2}\left(x_{1}: x_{2}\right) x_{0}^{2}+\cdots\right) \\
+\left(Q_{d-1}\left(x_{1}: x_{2}\right) x_{0}+Q_{d-2}\left(x_{1}: x_{2}\right) x_{0}^{2}+\cdots\right) t \\
+\left(R_{d}\left(x_{1}: x_{2}\right)+R_{d-1}\left(x_{1}: x_{2}\right) x_{0}+R_{d-2}\left(x_{1}: x_{2}\right) x_{0}^{2}+\cdots\right) t^{2}+\cdots .
\end{gathered}
$$

$Q_{d}=0$ means that $\gamma$ is tangent to the line $C \mu^{2}+K \mu t$, with $K$ a degree$(d-1)$ curve. Notice that $P_{d-2}$ gives the divisor $C_{\mu}$ on $\mu$, and $Q_{d-1}$ gives $K_{\mu} . \gamma$ is transversal to $B$ at $C \mu^{2}$ if $K_{\mu} \ngtr C_{\mu}$, i.e., if $P_{d-2}$ does not divide $Q_{d-1}$ (see $\left.\S 3.1,(1)\right)$. Such $\gamma$ are tangent to all line-conditions and transversal to $B$; therefore $\operatorname{th}_{C \mu^{2}}(\mathscr{S}) \geq 2$. We have to show that $\operatorname{th}_{C \mu^{2}}(\mathscr{S}) \geq 3$ only if $\mu$ is tangent to $C$, and that $\operatorname{th}_{C \mu^{2}}(\mathscr{S}) \leq 3$ if $\mu$ is simply tangent to $C$ at exactly one smooth point.

Restricting to $l$ as above, $\gamma(t)$ now cuts the divisor

$$
\begin{aligned}
\gamma_{l}(t)= & \left(P_{d-2}\left(l_{1}: l_{2}\right) \alpha^{d-2} \beta^{2}+\cdots\right) \\
& +\left(Q_{d-1}\left(l_{1}: l_{2}\right) \alpha^{d-1} \beta+Q_{d-2}\left(l_{1}: l_{2}\right) \alpha^{d-2} \beta^{2}+\cdots\right) t \\
& +\left(R_{d}\left(l_{1}: l_{2}\right) \alpha^{d}+R_{d-1}\left(l_{1}: l_{2}\right) \alpha^{d-1} \beta+R_{d-2}\left(l_{1}: l_{2}\right) \alpha^{d-2} \beta^{2}+\cdots\right) t^{2}+\cdots .
\end{aligned}
$$

By the claim, $\gamma$ has contact with order at least 3 with the line-condition corresponding to $l$ if and only if

$$
Q_{d-1}\left(l_{1}: l_{2}\right)^{2}=4 R_{d}\left(l_{1}: l_{2}\right) P_{d-2}\left(l_{1}: l_{2}\right) ;
$$


this can be realized for all $l_{1}, l_{2}$ if and only if $P_{d-2}$ divides $Q_{d-1}^{2}$. Therefore, $\gamma$ is transversal to $B$ and meets all line-conditions with order at least 3 at $C \mu^{2}$ if and only if $P_{d-2}$ does not divide $Q_{d-1}$ but $P_{d-2}$ divides $Q_{d-1}^{2}$; i.e., if and only if $K_{\mu} \geq C_{\mu}$ but $K_{\mu}^{2} \geq C_{\mu}$. This can happen only if $C_{\mu}$ has a 'double' point, i.e., only if $\mu$ is tangent to $C$ : Lemma II(1) and (3) follow. We note in passing that in fact this conclusion follows from requiring $(*)$ for $2 d-1$ general lines (i.e., $2 d-1$ general pairs $l_{1}, l_{2}$ ).

To finish the proof of Lemma II we need to show that, in the hypothesis of (2), $\gamma$ cannot meet all line-conditions with order $\geq 4$ at $C \mu^{2}$. This follows from part (iii) of the claim: $\gamma$ meets all line-conditions with order at least 4 if and only if

$$
(* *) \quad 2 P_{d-2}^{2} S_{d}-P_{d-2} Q_{d-1} R_{d-1}+2 P_{d-2} Q_{d-2} R_{d}-P_{d-3} Q_{d-1} R_{d}=0 ;
$$

but the first three terms in this sum have multiplicity at least 2 at the double point in $C_{\mu}$, while the last has multiplicity $1\left(P_{d-3}\right.$ cannot vanish there since we are assuming $C$ to be smooth at all intersections with $\mu$ ), so this cannot occur.

\section{THE BLOW-UPS}

The general plan is to blow-up the support $S$ of the intersection of all lineconditions, then the support of the intersection of their proper transforms. As remarked in $\S 1$, for our purposes we actually need only deal with the component $B$ of $S$ consisting of all curves containing a 'double line'; and in fact we are interested in analyzing the situation above nonsingular points of $B$ (cf. Lemma 1.3 in $\S 1$ ).

3.1. The first blow-up. As above, we denote by $B$ the subset of $\mathbb{P}^{N}$ formed by curves $C \mu^{2}$ containing a 'double line'; we will first blow-up $\mathbb{P}^{N}$ along $B$. Let $B^{\circ} \subset B$ be the open subset of $B$ consisting of all $C \mu^{2}$ with $C$ reduced and not containing $\mu$; recall that $B^{\circ}$ is smooth (cf. Lemma 1.1 in $\S 1$ ). Also, if $\mu \not \subset X$, denote by $X_{\mu}$ the divisor cut by the curve $X$ on the line $\mu$. The reader will easily check the following facts:

(1) The tangent space in $\mathbb{P}^{N}$ to $B$ at a point $C \mu^{2} \in B^{\circ}$ consists of all $K \mu \in \mathbb{P}^{N}$ with $K_{\mu} \geq C_{\mu}$ or $\mu \subset K$.

(2) Let $L$ be the line-condition in $\mathbb{P}^{N}$ corresponding to a line $l$. For $C \mu^{2} \in$ $B^{\circ}$ and $l$ general, $L$ is nonsingular at $C \mu^{2}$, and the tangent space in $\mathbb{P}^{N}$ to $L$ at $C \mu^{2}$ consists of all $X \in \mathbb{P}^{N}$ with $l \cap \mu \in X$.

In particular, it follows from (2) that the intersection of the tangent spaces of all line-conditions at a point $C \mu^{2} \in B^{\circ}$ consists of all $X \in \mathbb{P}^{N}$ that contain $\mu$ : indeed, a curve of degree $d$ cutting a line in more than $d$ points must contain it.

Let then $V_{1} \stackrel{\pi_{1}}{\rightarrow} \mathbb{P}^{N}$ be the blow-up of $\mathbb{P}^{N}$ along $B$, and call $E_{1}$ the exceptional divisor. Call 'point-' and 'line-conditions' in $V_{1}$ the proper transforms of the conditions in $\mathbb{P}^{N}$. As seen in $\S 1$, we need to analyze the blow-up over $B^{\circ}$.

Consider $\pi^{-1}\left(B^{\circ}\right)$, the subset of $E_{1}$ lying over $B^{\circ}$. As $B^{\circ}$ is smooth, $\pi^{-1}\left(B^{\circ}\right) \rightarrow B^{\circ}$ is a projective bundle: specifically, if $\mathscr{N}$ denotes the normal bundle to $B^{\circ}$ in $\mathbb{P}^{N}$, then $\pi^{-1}\left(B^{\circ}\right)=\mathbb{P}(\mathcal{N})$. So a point in $\pi^{-1}\left(B^{\circ}\right)$ is a normal direction to $B$ centered at a point $C \mu^{2} \in B^{\circ}$. 
Proposition 3.1. Denote by $\mathscr{S}_{1}$ the intersection of all line-conditions in $V_{1}$. Then $\mathscr{S}_{1} \cap \pi^{-1}\left(B^{\circ}\right)$ is supported on a $\mathbb{P}^{d-3}$-bundle $B_{1}^{\circ}$ over $B^{\circ}$.

Specifically (as we will see in the proof), the immersions $\operatorname{Sym}^{d-1} \check{\mathbb{V}} \hookrightarrow \operatorname{Sym}^{d} \check{\bigvee}$ given by multiplication by linear forms determine naturally a rank- $(d-2)$ subbundle $\mathscr{G}$ of $\mathscr{N}$, and $B_{1}^{\circ}=\mathbb{P}(\mathscr{G})$.

Proof. Call $B_{1}^{\circ}$ the support of $\mathscr{S}_{1} \cap \pi^{-1}\left(B^{\circ}\right)$. By (2) above, $\mathscr{S}_{1}$ intersects the fiber over $C \mu^{2} \in B^{\circ}$ along normal directions to $B$ lying in the space of curves $X \in \mathbb{P}^{N}$ containing $\mu$. These directions (i.e., the fiber of $B_{1}^{\circ}$ over each $C \mu^{2}$ ) form a $\mathbb{P}^{d-3}$; in fact, $B_{1}^{\circ}$ is the projectivization $\mathbb{P}(\mathscr{G})$ of a rank$(d-2)$ subbundle of $\mathscr{N}$. To show this (and to collect information which we will use in $\S 4.2$ ), recall from $\S 1$, proof of Lemma 1.1 , that $B^{\circ}$ is isomorphic to an open set in $\mathbb{P}^{(d-2)(d+1) / 2} \times \check{\mathbb{P}}^{2}$, via $(C, \mu) \mapsto C \mu^{2}$. Denote by $\mathscr{O}_{1}(1)$ (resp. $\left.\mathscr{O}_{2}(1)\right)$ the pull-back to $B^{\circ}$ of $\mathscr{O}(1)$ from the first (resp. second) factor of $\mathbb{P}^{(d-2)(d+1) / 2} \times \check{\mathbb{P}}^{2}$. The Euler sequence giving the tangent bundle to $\mathbb{P}^{N}$,

$$
0 \rightarrow \mathscr{O}_{\mathbb{P}^{N}} \longrightarrow \operatorname{Sym}^{d} \check{\mathbb{V}} \otimes \mathscr{O}_{\mathbb{P}^{N}}(1) \rightarrow T \mathbb{P}^{N} \rightarrow 0,
$$

pulls-back on $B^{\circ}$ to

$$
\left.0 \longrightarrow \mathscr{O}_{B^{\circ}} \longrightarrow \operatorname{Sym}^{d} \check{\mathbb{V}} \otimes \mathscr{O}_{1}(1) \otimes \mathscr{O}_{2}(2) \longrightarrow T \mathbb{P}^{N}\right|_{B^{\circ}} \longrightarrow 0 .
$$

Now, the immersion $\operatorname{Sym}^{d-1} \check{\bigvee} \otimes \mathscr{O}_{2}(-1) \hookrightarrow \mathrm{Sym}^{d} \check{\mathrm{V}}$ gives an immersion

$$
\operatorname{Sym}^{d-1} \check{\mathbb{V}} \otimes \mathscr{O}_{1}(1) \otimes \mathscr{O}_{2}(1) \hookrightarrow \operatorname{Sym}^{d} \check{\mathbb{V}} \otimes \mathscr{O}_{1}(1) \otimes \mathscr{O}_{2}(2)
$$

and determines a subbundle $\mathscr{G}^{\prime}$ of $\left.T \mathbb{P}^{N}\right|_{B^{\circ}}$ containing $T B^{\circ}$, and hence a subbundle $\mathscr{G}$ of $\mathscr{N}$. The fiber of $\mathscr{G}^{\prime}$ over $C \mu^{2} \in B^{\circ}$ is the tangent space at $C \mu^{2}$ to the set of curves of degree $d$ containing $\mu$, therefore (by the description of $B_{1}^{\circ}$ given above), $B_{1}^{\circ}=\mathbb{P}(\mathscr{G})$.

As seen above, the fiber of $B_{1}^{\circ}$ over $C \mu^{2}$ consists of the $(d-3)$-dimensional projective space of normal directions to $B$ centered at $C \mu^{2}$ and lying in the subspace of $\mathbb{P}^{N}$ formed by curves containing $\mu$. Call $\{K\}_{C \mu^{2}}$ the point in $B_{1}^{\circ}$ determined by the line $C \mu^{2}+t K \mu$ (parametrized by $t$ ), where $K$ is a degree- $(d-1)$ curve. Notice that for $K$ to determine a point of $B_{1}^{\circ}$, i.e., for $C \mu^{2}+t K \mu$ to determine a normal direction to $B$, we must have (by (1) above) $K_{\mu} \geq C_{\mu}$.

Now each point $p$ in $C \cap \mu$ determines a hyperplane in the fiber of $B_{1}^{\circ}$ over $C \mu^{2}$; namely the hyperplane consisting of all $\{K\}_{C \mu^{2}}$ with $p \in K$. If $\mu$ and $C$ intersect transversally, then $\mu \cap C$ consists of $d-2$ distinct points, and the $d-2$ corresponding hyperplanes in the fiber have empty intersection (because $K_{\mu} ¥ C_{\mu}$ for all $\left.\{K\}_{C \mu^{2}} \in B_{1}^{\circ}\right)$.

If on the other hand $\mu$ is tangent to $C$ (at one point), the $d-3$ points of the intersection of $\mu$ and $C$ determine $d-3$ hyperplanes in the fiber, intersecting at exactly one point. This determines a subset $T_{1}^{\circ} \subset B_{1}^{\circ}$, mapping bijectively onto the set $T^{\circ}=\left\{C \mu^{2} \in B^{\circ}\right.$ s.t. $\mu$ is tangent to $\left.C\right\}$. Equivalently, $T_{1}^{\circ}$ consists of all $\{K\}_{C \mu^{2}} \in B^{\circ}$ with $K_{\mu} \nsupseteq C_{\mu}, K_{\mu}^{2} \geq C_{\mu}$. This set will play an 
important role in the following: by Lemma 1.3 in $\S 1, T^{\circ}$ is disjoint from the intersection of $N-2 d+1$ general point-conditions, and meets the intersection of $N-2 d$ general point-conditions at finitely many points-in fact, finitely many points $C \mu^{2}$ with $\mu$ tangent to $C$ at a single smooth point.

Finally, we let $B_{1}, T_{1}$ be the closures of $B_{1}^{\circ}, T_{1}^{\circ}$ in $V_{1} . B_{1}$ will be the center for our second blow-up.

3.2. The second blow-up. Let $V_{2} \stackrel{\pi_{2}}{\rightarrow} V_{1}$ be the blow-up of $V_{1}$ along $B_{1}$, and call $E_{2}$ the new exceptional divisor. Again, we call 'point-conditions' and 'lineconditions' in $V_{2}$ the proper transforms of the conditions in $V_{1}$.

As before, we need only analyze the part of this blow-up lying over $B^{\circ}$. Now $B_{1}^{\circ}$ is nonsingular, therefore $\pi_{2}^{-1}\left(B_{1}^{\circ}\right) \subset E_{2}$ is a projective bundle over $B_{1}^{\circ}$ : denoting by $\mathscr{N}_{1}$ the normal bundle to $B_{1}^{\circ}$ in $V_{1}$, we have $\pi_{2}^{-1}\left(B_{1}^{\circ}\right)=\mathbb{P}\left(\mathscr{N}_{1}\right)$.

Denote by $\mathscr{E}_{1}$ the normal bundle to $B_{1}^{\circ}$ in $E_{1}$ : then the proper transform $\widetilde{E}_{1}$ of $E_{1}$ in $V_{2}$ intersects $\pi_{2}^{-1}\left(B_{1}^{\circ}\right)$ along $\mathbb{P}\left(\mathscr{E}_{1}\right) \subset \mathbb{P}\left(\mathscr{N}_{1}\right)$. The fiber of $\widetilde{E}_{1}$ over a point of $B_{1}^{\circ}$ is a hyperplane in the fiber of $E_{2}$; we first show that the line-conditions in $V_{2}$ do not meet anywhere along these hyperplanes:

Lemma 3.2. The intersection $\mathscr{S}_{2}$ of all line-conditions in $V_{2}$ is disjoint from $\pi_{2}^{-1}\left(B_{1}^{\circ}\right) \cap \widetilde{E}_{1}$.

Proof. Consider the Euler sequences for $B_{1}^{\circ}=\mathbb{P}(\mathscr{G})$ and $\pi_{1}^{-1}\left(B^{\circ}\right)=\mathbb{P}(\mathscr{N})$ (notations as in the proof of Proposition 3.1):

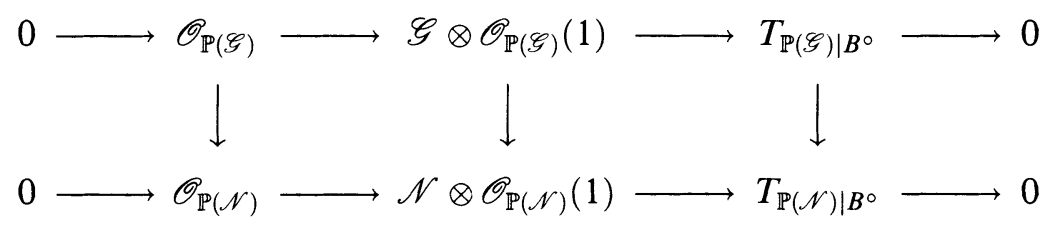

These give (with some abuse of notation)

$$
\mathscr{E}_{1}=T_{\mathbb{P}(\mathcal{N}) \mid B^{\circ}} / T_{\mathbb{P}(\mathscr{G}) \mid B^{\circ}}=(\mathscr{N} / \mathscr{G}) \otimes \mathscr{O}_{\mathbb{P}(\mathscr{G})}(1) .
$$

Recalling how $\mathscr{G}$ was obtained, we have

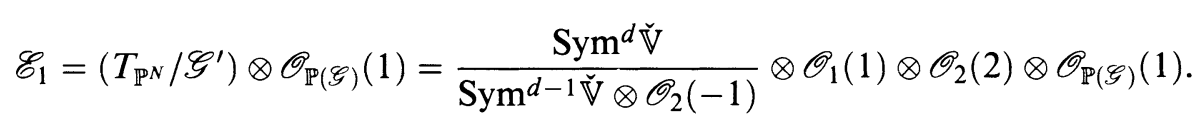

Let $p \in B_{1}^{\circ}$, mapping to $C \mu^{2} \in B^{\circ}$. The fiber of $\mathbb{P}\left(\mathscr{E}_{1}\right)$ over $p$ can be identified with $\mathbb{P}\left(\operatorname{Sym}^{d} \check{\mathbb{V}} / \operatorname{Sym}^{d-1} \check{\mathbb{V}}\right)$, where the inclusion $\operatorname{Sym}^{d-1} \check{\mathrm{V}} \hookrightarrow \operatorname{Sym}^{d} \check{\mathrm{V}}$ is given by multiplication by $\mu$.

Therefore $\widetilde{E}_{1} \cap \pi_{2}^{-1}(p)$ can be identified with the space of $d$-tuples of points over $\mu$; via this identification, the line-condition $L_{l}$ in $V_{2}$ corresponding to a general line $l$ contains a point in $\widetilde{E}_{1} \cap \pi_{2}^{-1}(p)$ if and only if $l$ goes through a point of the $d$-tuple. The assertion of the lemma follows from this.

Since the general line-condition in $V_{1}$ is nonsingular at a point $p \in B_{1}^{\circ}$, then $\pi_{2}^{-1}(p) \cap \mathscr{S}_{2}$ is (set-theoretically) the intersection of hyperplanes of the fiber of $\mathbb{P}\left(\mathscr{N}_{1}\right)$, and is therefore itself a linear space. By Lemma 3.2, this intersection 
misses a hyperplane in each fiber, hence it consists of at most one point over each $p \in B_{1}^{\circ}$.

Lemma 3.2 is the main tool in our next observation. Recall that, over $B^{\circ}$, the intersection $\mathscr{S}_{1}$ of all line-conditions in $V_{1}$ is supported on $B_{1}^{\circ}$. Also, we found a 'special' subvariety $T_{1}^{\circ} \subset B_{1}^{\circ}$ : denoting (as in $\S 1$ ) by $\{K\}_{C \mu^{2}}$ points of $B_{1}^{\circ}, T_{1}^{\circ}$ consists of all $\{K\}_{C \mu^{2}} \in B_{1}^{\circ}$ with $K_{\mu}^{2} \geq C_{\mu}$.

Lemma 3.3. (1) $\operatorname{th}_{p}\left(\mathscr{S}_{1}\right)=1$ if $p \in B_{1}^{\circ}-T_{1}^{\circ}$.

(2) $\operatorname{th}_{p}\left(\mathscr{S}_{1}\right) \geq 2$ if $p \in T_{1}^{\circ}$, and $\operatorname{th}_{p}\left(\mathscr{S}_{1}\right)=2$ if $p=\{K\}_{C \mu^{2}}$, with $\mu$ tangent to $C$ at a single smooth point.

Proof. The thickness of $\mathscr{S}_{1}$ is (vacuously) at least 1 at all $p \in B_{1}^{\circ}$; we have to show it is at most 1 outside of $T_{1}^{\circ}$, and precisely 2 on $T_{1}^{\circ}$ if $p$ maps to $C \mu^{2}$, $\mu$ tangent to $C$ at a single smooth point.

Let $p=\{K\}_{C \mu^{2}} \in B_{1}^{\circ}$. It follows from Lemma 3.2 that a nonsingular curve in $V_{1}$ tangent to $E_{1}$ and transversal to $B_{1}$ at $p$ must be transversal to the general line-condition in $V_{1}$ at $p$; indeed, otherwise the line-conditions in $V_{2}$ would intersect along $\widetilde{E}_{1} \cap E_{2}$ above $p$. Therefore, germs centered at $p$, transversal to $B_{1}$, and tangent to $E_{1}$ intersect $\mathscr{S}_{1}$ with multiplicity 1 at $p$. On the other hand, consider a smooth curve germ $\gamma(t)$ centered at $\{K\}_{C \mu^{2}}$ and transversal to $B_{1}$ and $E_{1}$; such a $\gamma$ maps down to a smooth curve germ in $\mathbb{P}^{N}$, centered at $C \mu^{2}$ and transversal to $B$. Then (1) and (2) follow from Lemma II by, e.g., [F, Theorem 12.4(a)].

The last lemma is the main ingredient for

Proposition 3.4. $\mathscr{S}_{2} \cap \pi_{2}^{-1}\left(B_{1}^{\circ}\right)$ is supported on a variety $B_{2}^{\circ}$ mapping bijectively onto $T_{1}^{\circ}$.

Proof. By Lemma 3.2, the intersection consists of at most one point in each fiber over $p \in B_{1}^{\circ}$. Now the line-conditions in $V_{2}$ cannot intersect above points $p \in B_{1}^{\circ}$ where $\operatorname{th}_{p}\left(\mathscr{S}_{1}\right)<2$, and they must intersect above $p$ if $\operatorname{th}_{p}\left(\mathscr{S}_{1}\right) \geq 2$ (since the line-conditions in $V_{1}$ share a normal direction to $B_{1}^{\circ}$ in this case). The statement then follows from the computation of $\operatorname{th}_{p}\left(\mathscr{S}_{1}\right)$ in Lemma 3.3.

In fact $B_{2}^{\circ}$ is a section of $E_{2}$ over $T_{1}^{\circ}$; we set $\widetilde{V}=V_{2}$, and let $B_{2}$ be the closure of $B_{2}^{\circ}$ in $\widetilde{V}$. In $\S 4$, we will use $\widetilde{V}$ to compute $N_{d}(2 d-1)$ and $N_{d}(2 d)$. We summarize the results of this section in

Lemma III. Let $\widetilde{P}$ and $\widetilde{L}$ denote the classes of the general point- and lineconditions in $\widetilde{V}$, resp. Then

(1) $N_{d}(2 d-1)=\widetilde{P}^{N-2 d+1} \cdot \widetilde{L}^{2 d-1}$, and

(2) $N_{d}(2 d)=\widetilde{P}^{N-2 d} \cdot \widetilde{L}^{2 d}-c$, where $c$ is a (positive) contribution due to finitely many points in $B_{2}^{\circ}$.

Proof. This follows from Lemma I, Lemma 1.3, and Proposition 3.4: the intersection of $N-2 d+1$ general point-conditions in $\widetilde{V}$ is disjoint from $\mathscr{S}_{2}$ (giving (1)), while the intersection of $N-2 d$ general point-conditions in $\widetilde{V}$ meets $\mathscr{S}_{2}$ at finitely many points of $B_{2}^{\circ}$, so that $\widetilde{P}^{N-2 d} \cdot \widetilde{L}^{2 d}$ is the sum of $N_{d}(2 d)$ and of a positive contribution $c$. 


\section{TWO CHARACTERISTIC NUMBERS FOR ALL DEGREES}

In this section we apply the construction in $\S 3$ to prove

Theorem IV. Denote by $N_{d}(k)$ the number of smooth plane curves of degree $d$ tangent to $k$ lines and containing $\frac{d(d+3)}{2}-k$ points in general position. Then

(1) $N_{d}(2 d-1)=(2 d-2)^{2 d-1}-2^{d-3} d(d-1)\left(d^{2}-d+2\right)$.

(2) $N_{d}(2 d)=(2 d-2)^{2 d}-2^{d-4} d(d-1)\left(8 d^{4}-21 d^{3}+19 d^{2}-20 d+32\right)$.

Remark. By Bézout's theorem in $\mathbb{P}^{N}$ and Lemma 1.3, $(2 d-2)^{2 d-1}$ must be the sum of $N_{d}(2 d-1)$ and of a contribution given by the $\frac{d(d-1)\left(d^{2}-d+2\right)}{8}$ nonreduced curves $C \mu^{2} \in \mathbb{P}^{N}$ that contain $N-2 d+1$ general points $p_{1}, \ldots$, $p_{N-2 d+1}$. Suppose $C \mu^{2}$ is one such curve, and that $p_{1}, p_{2} \in \mu$, and $p_{3}, \ldots$, $p_{N-2 d+1} \in C$. Now $d$ general line-conditions and the point-conditions corresponding to $p_{3}, \ldots, p_{N-2 d+1}$ intersect in a $(d+1)$-dimensional variety $Z \subset$ $\mathbb{P}^{N}$ nonsingular at $C \mu^{2}$ : their tangent hyperplanes intersect on the $(d+1)$ dimensional subspace formed by curves containing $p_{3}, \ldots, p_{N-2 d+1}$ and $d$ points $q_{1}, \ldots, q_{d}$ on $\mu$ (cf. (2) in $\S 3.1$ ). A general line-condition will now intersect $Z$ in a divisor nonsingular at $C \mu^{2}$, whose tangent space at $C \mu^{2}$ is the set of all curves containing $\mu$ (a degree- $d$ curve containing $d+1$ aligned points must contain the line through them); the same holds for the point-conditions corresponding to $p_{1}$ and $p_{2}$.

Therefore the contribution of $C \mu^{2}$ to the total intersection number is the contribution of an isolated point of the intersection of $d+1$ nonsingular divisors all tangent to one another in a $(d+1)$-dimensional variety. Such a contribution is at least $2^{d}$ [F, Example 8.2.2]. Part (1) of Theorem IV implies that the contribution of each nonreduced curve in this enumerative problem is precisely $2^{d}$.

The rest of this section is devoted to deriving Theorem IV from Lemma III. We will use a formula relating intersections under blow-ups (see [A, §2] for the proof of a statement implying this):

Proposition. Let $V$ be a smooth $n$-dimensional variety, $B \stackrel{i}{\hookrightarrow} V$ a smooth subvariety, $X_{1}, \ldots, X_{n}$ divisors of $V$, and denote by $e_{B} X$ the multiplicity of $X$ along $B$. Let $\widetilde{V} \stackrel{\pi}{\rightarrow} V$ be the blow-up of $V$ along $B$, and $\widetilde{X}_{1}, \ldots, \widetilde{X}_{n}$ the proper transforms of $X_{1}, \ldots, X_{n}$. Then

$$
\pi_{*}\left(\widetilde{X}_{1} \cdots \widetilde{X}_{n}\right)=X_{1} \cdots X_{n}-i_{*}\left\{\frac{\prod_{j}\left(e_{B} X_{j}[B]+i^{*}\left[X_{j}\right]\right)}{c\left(N_{B} V\right)}\right\}_{0}
$$

in $A_{0}\left(\bigcap_{j} X_{j}\right)$ (here $\{\alpha\}_{0}$ denotes the 0-dimensional component of the class $\alpha$ ).

If e.g., $B$ and $V$ are complete, we can use this formula to. compare the degrees of the intersection of $X_{1}, \ldots, X_{n}$ in $V$ and of $\widetilde{X}_{1}, \ldots, \widetilde{X}_{n}$ in $\widetilde{V}$. We want to apply this formula to the two blow-ups examined in $\S 3$; a little extra care has to be taken since we studied the blow-ups only over certain (open) subsets of the varieties involved. Suppose then that $V$ is a complete $n$-dimensional variety, $i: B \rightarrow V$ and $\pi: \widetilde{V} \rightarrow V$ are proper maps, $X_{1}, \ldots, X_{n}$ are divisors in $V$, and $\widetilde{X}_{1}, \ldots, \widetilde{X}_{n}$ are divisors in $\widetilde{V}$. Suppose that there exists a nonsingular dense open set $V^{\circ} \subset V$ such that $B^{\circ}=i^{-1}\left(V^{\circ}\right) \hookrightarrow V^{\circ}$ is an 
embedding of smooth varieties, $\pi^{-1}\left(V^{\circ}\right) \rightarrow V^{\circ}$ is the blow-up of $V^{\circ}$ along $B^{\circ}, \tilde{X}_{j} \cap \pi^{-1}\left(V^{\circ}\right)$ are the proper transforms of $X_{j} \cap V^{\circ}$, and $X_{1} \cap \cdots \cap X_{n} \subset V^{\circ}$, $\widetilde{X}_{1} \cap \cdots \cap \widetilde{X}_{n} \subset \pi^{-1}\left(V^{\circ}\right)$ (i.e., the situation pulls-back to that of the proposition when restricting to $V^{\circ}$ ). Then, denoting by $e_{B} X$ the multiplicity of $X$ along $B^{\circ}$,

$$
\int_{\widetilde{V}} \tilde{X}_{1} \cdots \widetilde{X}_{n}=\int_{V} X_{1} \cdots X_{n}-\int_{B} \frac{\prod_{j}\left(e_{B} X_{j}[B]+i^{*}\left[X_{j}\right]\right)}{c\left(N_{B} V\right)},
$$

where $c\left(N_{B} V\right)$ is any class that pulls-back to $c\left(N_{B^{\circ}} V^{\circ}\right)$ on $B^{\circ}$.

This follows from the proposition above, since all the terms are sums of local contributions. As the reader will check, Lemma 1.3 and our choices guarantee that the hypotheses are satisfied at both stages of our computation.

4.1. The first blow-up. We apply $(*)$ to

$$
\begin{gathered}
\mathbb{P}^{(d-2)(d+1) / 2} \times \check{\mathbb{P}}^{2} \stackrel{i}{\longrightarrow} \mathbb{P}^{d(d+3) / 2} \\
(C, \mu) \mapsto C \mu^{2}
\end{gathered}
$$

(cf. the proof of Lemma 1.1). Keeping the notation as in $\S 3.1$, the image of this map is $B$; we analyzed the blow-up over $B^{\circ}$, which is identified via this map with the subset $B^{\circ} \subset \mathbb{P}^{(d-2)(d+1) / 2} \times \breve{\mathbb{P}}^{2}$ consisting of the pairs $(C, \mu)$ with $C$ reduced and not containing $\mu$. If $P$ and $L$ denote point- and line-conditions in $\mathbb{P}^{N}$, resp., then $e_{B} P=0$ and $e_{B} L=1$ (line-conditions are generically smooth along $B$, cf. (2) in $\S 3.1)$. The Chow ring of $\mathbb{P}^{(d-2)(d+1) / 2} \times \breve{\mathbb{P}}^{2}$ is generated by the pull-backs $l, m$ of the hyperplane classes from the factors, with the relations $l^{i} m^{j}=0$ if $i>\frac{(d-2)(d+1)}{2}$ or $j>2$, and $\int l^{(d-2)(d+1) / 2} m^{2}=1$. Also, $i^{*} P=l+2 m$ and $i^{*} L=(2 d-2) l+(4 d-4) m$. Denoting by $P_{1}$ and $L_{1}$ the classes of the line-conditions in $V_{1}$, Bézout's theorem and $(*)$ give (for $k \leq 2 d$ )

$$
P_{1}^{N-k} \cdot L_{1}^{k}=(2 d-2)^{k}-\int(l+2 m)^{N-k} \frac{(1+(2 d-2) l+(4 d-4) m)^{k}}{c\left(N_{B} \mathbb{P}^{N}\right)},
$$

where $c\left(N_{B} \mathbb{P}^{N}\right)$ is any class on $\mathbb{P}^{(d-2)(d+1) / 2} \times \check{\mathbb{P}}^{2}$ restricting to $c\left(N_{B^{\circ}} \mathbb{P}^{N}\right)$ on $B^{\circ}$. Such is

$$
\begin{aligned}
\frac{i^{*} c\left(T \mathbb{P}^{N}\right)}{c\left(T \mathbb{P}^{(d-2)(d+1) / 2} \times \check{\mathbb{P}}^{2}\right)} & =\frac{(1+l+2 m)^{\left(\begin{array}{c}
d+2 \\
2
\end{array}\right)}}{(1+l)^{\left(\begin{array}{c}
d \\
2
\end{array}\right)}(1+m)^{3}} \\
& =1+(2 d+1) l+\left(d^{2}+3 d-1\right) m+\cdots
\end{aligned}
$$

(only these terms are relevant for $k \leq 2 d$ ). Therefore,

and

$$
\begin{aligned}
& P_{1}^{N-2 d+1} \cdot L_{1}^{2 d-1}=(2 d-2)^{2 d-1}-\int(l+2 m)^{\left(\begin{array}{l}
d \\
2
\end{array}\right)+1} \\
& =(2 d-2)^{2 d-1}-4\left(\begin{array}{c}
\left(\begin{array}{c}
d \\
2
\end{array}\right)+1 \\
2
\end{array}\right) \text {, }
\end{aligned}
$$

$$
\begin{gathered}
P_{1}^{N-2 d} \cdot L_{1}^{2 d}=(2 d-2)^{2 d}-\int(l+2 m)^{\left(\begin{array}{c}
d \\
2
\end{array}\right)} \frac{(1+2 d(2 d-2) l+2 d(4 d-4) m)}{1+(2 d+1) l+\left(d^{2}+3 d-1\right) m} \\
=(2 d-2)^{2 d}-\int(l+2 m)^{\left(\begin{array}{l}
d \\
2
\end{array}\right)}\left(\left(4 d^{2}-6 d-1\right) l+\left(7 d^{2}-11 d+1\right) m\right) .
\end{gathered}
$$


4.2. The second blow-up; $N_{d}(2 d-1)$. The center of the second blow-up is the closure of $B_{1}^{\circ}$, a $\mathbb{P}^{d-3}$-bundle over $B^{\circ}$. More precisely (see Proposition 3.1) the immersion $\operatorname{Sym}^{d-1} \check{\mathrm{V}} \otimes \mathscr{O}_{1}(1) \otimes \mathscr{O}_{2}(1) \hookrightarrow \operatorname{Sym}^{d} \check{\mathbb{V}} \otimes \mathscr{O}_{1}(1) \otimes \mathscr{O}_{2}(2)$ determines a subbundle $\mathscr{G}^{\prime}$ of $\left.T \mathbb{P}^{N}\right|_{B^{\circ}}$ containing $T B^{\circ}$, therefore a subbundle $\mathscr{G}$ of the normal bundle $\mathscr{N}$ to $B^{\circ}$ in $\mathbb{P}^{N}$, and $B_{1}^{\circ}=\mathbb{P}(\mathscr{G})$.

Now call $\overline{\mathbb{P}(\mathscr{G})}$ any compactification of $\mathbb{P}(\mathscr{G})$ filling the diagram

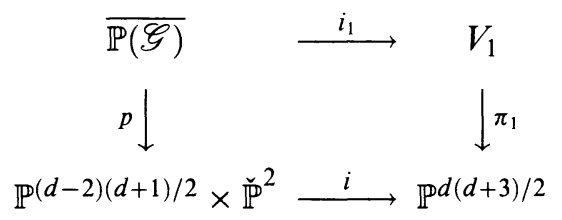

with $i_{1}$ proper, identifying $i_{1}^{-1} B_{1}^{\circ}$ with $B_{1}^{\circ}$, and $B_{1}^{\circ} \stackrel{p}{\longrightarrow} B^{\circ}$ with the bundle $\mathbb{P}(\mathscr{G})$ defined above. Also, denote by $l, m$ the pull-backs of $l, m$ via $p$, and by $e$ the class of the 'universal line bundle' (i.e., the pull-back of the class of $E_{1}$ via $\left.i_{1}\right)$.

\section{Lemma 4.1.}

$$
p_{*} e^{j}= \begin{cases}0, & j<d-3, \\ (-1)^{d-1}, & j=d-3, \\ (-1)^{d-1}\left(d l+\frac{d^{2}+d-6}{2} m\right), & j=d-2 .\end{cases}
$$

Proof. The codimension of the complement of $B^{\circ}$ in $\mathbb{P}^{(d-2)(d+1) / 2} \times \check{\mathbb{P}}^{2}$ is $\geq 2$, so it is enough to observe that, over $B^{\circ}, \sum_{j} e^{j}$ pushes forward to the Segre class $s(\mathscr{G})$ of $\mathscr{G}$ : tracing the definition of $\mathscr{G}$, we have

$$
\begin{aligned}
s(\mathscr{G}) & =\frac{c\left(T \mathbb{P}^{(d-2)(d+1) / 2} \times \check{\mathbb{P}}^{2}\right)}{c\left(\mathscr{G}^{\prime}\right)}=\frac{c\left(T \mathbb{P}^{(d-2)(d+1) / 2}\right) c\left(\check{\mathbb{P}}^{2}\right)}{c\left(\operatorname{Sym}^{d-1} \check{\mathbb{V}} \otimes \mathscr{O}_{1}(1) \otimes \mathscr{O}_{2}(1)\right)} \\
& =\frac{(1+l)^{\left(\begin{array}{c}
d \\
2
\end{array}\right)}(1+m)^{3}}{(1+l+m)^{\left(\begin{array}{c}
d+1 \\
2
\end{array}\right)}}=1-d l-\frac{d^{2}+d-6}{2} m+\cdots .
\end{aligned}
$$

Denote (as above) by $P_{1}$ and $L_{1}$ the classes of the point- and line-conditions in $V_{1}$, and by $\widetilde{P}$ and $\widetilde{L}$ the classes of the conditions in $\widetilde{V}=V_{2}$, resp. We have $e_{B_{1}} P_{1}=0, e_{B_{1}} L_{1}=1$, and $i_{1}^{*} P_{1}=l+2 m, i_{1}^{*} L_{1}=(2 d-2) l+(4 d-4) m-e$, and $i_{1}^{*} E_{1}=e$. Therefore $(*)$ gives (for $k \leq 2 d$ )

$$
\widetilde{P}^{N-k} \cdot \widetilde{L}^{k}=P_{1}^{N-k} \cdot L_{1}^{k}-\int(l+2 m)^{N-k} \frac{(1+(2 d-2) l+(4 d-4) m-e)^{k}}{c\left(N_{B_{1}} V_{1}\right)},
$$

where $c\left(N_{B_{1}} V_{1}\right)$ is any class on $\overline{\mathbb{P}(\mathscr{G})}$ restricting to $c\left(N_{B_{1}^{\circ}} V_{1}\right)$ on $B_{1}^{\circ}$. Now $N_{B_{1}^{\circ}} V_{1}$ is an extension of $N_{E_{1}} V_{1}$ and $\mathscr{E}_{1}=N_{B_{1}^{\circ}} E_{1}$ (notation as in $\S 3.2$ ); we computed $\mathscr{E}_{1}$ in the proof of Lemma 3.2, getting

$$
\mathscr{E}_{1}=\frac{\operatorname{Sym}^{d} \check{\mathbb{V}}}{\operatorname{Sym}^{d-1} \check{\mathbb{V}} \otimes \mathscr{O}_{2}(-1)} \otimes \mathscr{O}_{1}(1) \otimes \mathscr{O}_{2}(2) \otimes \mathscr{O}_{\mathbb{P}(\mathscr{G})}(1) .
$$

Putting all this together, we can set

$$
c\left(N_{B_{1}} V_{1}\right)=(1+e) \frac{(1+l+2 m-e)^{\left(\begin{array}{c}
d+2 \\
2
\end{array}\right)}}{(1+l+m-e)^{\left(\begin{array}{c}
d+1 \\
2
\end{array}\right)}} .
$$


Hence in order to apply $(*)$ we have to evaluate the degree on $\overline{P(\mathscr{G})}$ of

$$
(l+2 m)^{N-k} \frac{(1+(2 d-2) l+(4 d-4) m-e)^{k}(1+l+m-e)^{\left(\begin{array}{c}
d+1 \\
2
\end{array}\right)}}{(1+e)(1+l+2 m-e)^{\left(\begin{array}{c}
d+2 \\
2
\end{array}\right)}}
$$

for $k=2 d-1,2 d$, or equivalently the degree on $\mathbb{P}^{(d-2)(d+1) / 2} \times \check{\mathbb{P}}^{2}$ of

$$
(l+2 m)^{N-k} p_{*} \frac{(1+(2 d-2) l+(4 d-4) m-e)^{k}(1+l+m-e)^{\left(\begin{array}{c}
d+1 \\
2
\end{array}\right)}}{(1+e)(1+l+2 m-e)^{\left(\begin{array}{c}
d+2 \\
2
\end{array}\right)} .}
$$

For $k=2 d-1$, the only relevant term comes from the push-forward via $p$ of the term of degree $d-3$ in

$$
\frac{(1+(2 d-2) l+(4 d-4) m-e)^{2 d-1}(1+l+m-e)^{\left(\begin{array}{c}
d+1 \\
2
\end{array}\right)}}{(1+e)(1+l+2 m-e)^{\left(\begin{array}{c}
d+2 \\
2
\end{array}\right)}} .
$$

By the projection formula and Lemma 4.1 , the only degree- $(d-3)$ monomial in $l, m, e$ with nonzero push-forward is $e^{d-3}$; thus the only relevant term is the term of degree $d-3$ in

$$
\frac{(1-e)^{2 d-1}(1-e)^{\left(\begin{array}{c}
d+1 \\
2
\end{array}\right)}}{(1+e)(1-e)^{\left(\begin{array}{c}
d+2 \\
2
\end{array}\right)}}=\frac{(1-e)^{d-2}}{1+e} .
$$

This is $(-1)^{d-1}\left(2^{d-2}-1\right) e^{d-3}$, therefore Lemma 4.1 gives

$$
\begin{aligned}
& \int(l+2 m)^{\left(\begin{array}{c}
d \\
2
\end{array}\right)+1} p_{*} \frac{(1+(2 d-2) l+(4 d-4) m-e)^{2 d-1}(1+l+m-e)^{\left(\begin{array}{c}
d+1 \\
2
\end{array}\right)}}{(1+e)(1+l+2 m-e)^{\left(\begin{array}{c}
d+2 \\
2
\end{array}\right)}} \\
& =\int\left(2^{d-2}-1\right)(l+2 m)^{\left(\begin{array}{c}
d \\
2
\end{array}\right)+1}=4\left(2^{d-2}-1\right)\left(\begin{array}{c}
\left(\begin{array}{c}
d \\
2
\end{array}\right)+1 \\
2
\end{array}\right) \text {. }
\end{aligned}
$$

This computation, Lemma III at the end of $\S 3$, and $P_{1}^{N-2 d+1} \cdot L_{1}^{2 d-1}$ as obtained in $\S 4.1$ yield

$$
N_{d}(2 d-1)=\widetilde{P}^{N-2 d+1} \cdot \widetilde{L}^{2 d-1}=(2 d-2)^{2 d-1}-2^{d}\left(\begin{array}{c}
\left(\begin{array}{c}
d \\
2
\end{array}\right)+1 \\
2
\end{array}\right),
$$

which is part (1) of Theorem IV.

For $k=2 d$, the computation runs along the same lines. We now need the term of degree $d-2$ in

$$
\frac{(1+(2 d-2) l+(4 d-4) m-e)^{2 d}(1+l+m-e)^{\left(\begin{array}{c}
d+1 \\
2
\end{array}\right)}}{(1+e)(1+l+2 m-e)^{\left(\begin{array}{c}
d+2 \\
2
\end{array}\right)}} ;
$$

by Lemma 4.1 , the only monomials with nonzero push-forward are $e^{d-2}$, $l e^{d-3}, m e^{d-3}$. As above, the coefficient of $e^{d-2}$ in the expression is the coefficient of $e^{d-2}$ in

$$
\frac{(1-e)^{2 d}(1-e)^{\left(\begin{array}{c}
d+1 \\
2
\end{array}\right)}}{(1+e)(1-e)^{\left(\begin{array}{c}
d+2 \\
2
\end{array}\right)}}=\frac{(1-e)^{d-1}}{1+e}
$$


i.e., $(-1)^{d}\left(2^{d-1}-1\right)$. One computes similarly the coefficients of $l e^{d-3}, m e^{d-3}$; the result is that the only relevant term in the expression above is

$$
(-1)^{d}\left(\left(2^{d-1}-1\right) e^{d-2}-\left(2^{d-2}-1\right)\left[\left(4 d^{2}-5 d-1\right) l+\frac{15 d^{2}-21 d-4}{2} m\right]\right) \text {. }
$$

Applying Lemma 4.1, the push-forward of this class is

$$
\begin{gathered}
-\left(2^{d-1}-1\right)\left(d l+\frac{d^{2}+d-6}{2} m\right)+\left(2^{d-2}-1\right) \\
\quad \times\left[\left(4 d^{2}-5 d-1\right) l+\frac{15 d^{2}-21 d-4}{2} m\right] \\
=2^{d-3}\left(\left(4 d^{2}-9 d-1\right) l+\left(13 d^{2}-23 d+8\right) m\right) \\
+\frac{4 d^{2}-7 d-1}{2} l+\left(7 d^{2}-11 d+1\right) m,
\end{gathered}
$$

hence applying $(*)$ and the computation of $P_{1}^{N-2 d} \cdot L_{1}^{2 d}$ in $\S 4.1$ yields $\widetilde{P}^{N-2 d}$. $\widetilde{L}^{2 d}$ :

$$
\begin{aligned}
& (2 d-2)^{2 d}-\int(l+2 m)^{\left(\begin{array}{c}
d \\
2
\end{array}\right)}\left(2^{d-2}\left(4 d^{2}-7 d-1\right) l+2^{d-3}\left(13 d^{2}-23 d+8\right) m\right) \\
& =(2 d-2)^{2 d}-4\left(\begin{array}{c}
\left(\begin{array}{c}
d \\
2
\end{array}\right) \\
2
\end{array}\right) 2^{d-2}\left(4 d^{2}-7 d-1\right)-2\left(\begin{array}{l}
d \\
2
\end{array}\right) 2^{d-3}\left(13 d^{2}-23 d+8\right),
\end{aligned}
$$

or

$$
\widetilde{P}^{N-2 d} \cdot \widetilde{L}^{2 d}=(2 d-2)^{2 d}-2^{d-3} d(d-1)\left(4 d^{4}-11 d^{3}+11 d^{2}-8 d+10\right) .
$$

In the next section, we use this result and Lemma III(2) from $\S 3$ to conclude the proof of Theorem IV.

4.3. $N_{d}(2 d)$. By Lemma III $(2)$ and the result in $\S 4.2$, in $\widetilde{V}$

$$
\widetilde{P}^{N-2 d} \cdot \widetilde{L}^{2 d}=(2 d-2)^{2 d}-2^{d-3} d(d-1)\left(4 d^{4}-11 d^{3}+11 d^{2}-8 d+10\right)
$$

is the sum of $N_{d}(2 d)$ and of a contribution due to finitely many points of $B_{2}$. More precisely, let $\widetilde{P}_{1}, \ldots, \widetilde{P}_{N-2 d}$ be general point-conditions, and let $\widetilde{L}_{1}, \ldots, \widetilde{L}_{2 d}$ be general line-conditions in $\widetilde{V}=V_{2}$ (notation as in $\S 3$ ). We have shown that $\widetilde{P}_{1} \cap \cdots \cap \widetilde{P}_{N-2 d} \cap \widetilde{L}_{1} \cap \cdots \cap \widetilde{L}_{2 d}$ consists of $N_{d}(2 d)$ 'good' points corresponding to smooth degree- $d$ curves satisfying the conditions, and finitely many 'bad' points in $B_{2}^{\circ}$. The intersection is transversal at the good points; we have to evaluate the contribution to $\widetilde{P}_{1} \ldots \widetilde{P}_{N-2 d} \cdot \widetilde{L}_{1} \ldots \widetilde{L}_{2 d}$ due to points of $B_{2}^{\circ}$. Our plan is the following: we will basically produce explicitly the (scheme-theoretic) component $\mathscr{B}_{2}$ of $\widetilde{L}_{1} \cap \cdots \cap \widetilde{L}_{2 d}$ that contains the 'bad' points. $\mathscr{B}_{2}$ is supported on $B_{2} ;$ in fact, we will compute $\left[\mathscr{B}_{2}\right]=2^{d-4}\left[B_{2}\right]$. Then

$$
\widetilde{P}^{N-2 d} \cdot \widetilde{L}^{2 d}=N_{d}(2 d)+2^{d-4} \widetilde{P}_{1} \cdots \cdot \widetilde{P}_{N-2 d} \cdot B_{2} ;
$$

part (2) of Theorem IV follows by comparing this to the other expression for $\widetilde{P}^{N-2 d} \cdot \widetilde{L}^{2 d}$ obtained above.

Let $p \in B_{2}$ be a 'bad' point; by Lemma 1.3(2), $p$ maps down to a $C \mu^{2} \in B$ with $\mu$ tangent to $C$ at a single smooth point. We have to express $\mathscr{B}_{2}$ explicitly in a neighborhood of $p$; the main observation to this effect is 
Claim 1. $\mathscr{S}_{2} \subset E_{2}$ scheme-theoretically in a neighborhood of $p$.

Proof. Choose local parameters $\left\{f_{1}, \ldots, f_{N}\right\}$ for $V_{2}$ at $p$ such that $f_{1}=0$ is a local equation for $E_{2}$ at $p$ and $\left\{f_{2}, \ldots, f_{N}\right\}$ are local parameters for $E_{2}$ at $p$. Let $I_{p}\left(\mathscr{S}_{2}\right)$ be the ideal of $\mathscr{S}_{2}$ in the local ring for $V_{2}$ at $p$; since $\mathscr{S}_{2}$ is contained in $E_{2}$ set-theoretically in a neighborhood of $p$, there is a least integer $k$ such that $f_{1}^{k} \in I_{p}\left(\mathscr{S}_{2}\right)$. We claim that $k=1$. Indeed, consider the curve germ $\gamma$ defined by $\gamma(t)=(t, 0, \ldots, 0) . \gamma$ is transversal to $E_{2}$, and intersects $\mathscr{S}_{2}$ with multiplicity $k$ at 0 ; therefore it maps down to a curve germ $\pi_{2}(\gamma)$ transversal to $B_{1}$ at $\pi_{2}(p) \in T_{1}^{\circ}$, intersecting $\mathscr{S}_{1}$ with multiplicity $k+1$ at 0 . But the thickness of $\mathscr{S}_{1}$ at $\pi_{2}(p)$ is 2 (Lemma 3.3), so this implies $k=1$.

In fact this argument shows that, for $2 d$ general line-conditions $\widetilde{L}_{1}, \ldots$, $\widetilde{L}_{2 d}$, we have $\widetilde{L}_{1} \cap \cdots \cap \widetilde{L}_{2 d} \subset E_{2}$ scheme-theoretically in a neighborhood of $p$ (the inclusion holds set-theoretically, and the thickness of the intersection of the corresponding line-conditions in $V_{1}$ is 2 by the same argument used in $\S 3.3$ ); since there are only finitely many 'bad' points, for a general choice of lines the inclusion will hold in a neighborhood of all of them.

By the preceding observation, $\widetilde{L}_{1} \cap \cdots \cap \widetilde{L}_{2 d}=\left(\widetilde{L}_{1} \cap E_{2}\right) \cap \cdots \cap\left(\widetilde{L}_{2 d} \cap E_{2}\right)$ in a neighborhood of the bad points. This is useful because the $\widetilde{L}_{j} \cap E_{2}$ can be described very explicitly by mean of the computation in $\S 2$. Indeed, consider $C \mu^{2} \in B^{\circ} \subset \mathbb{P}^{N}$ and $\{K\}_{C \mu^{2}} \in B_{1}^{\circ}$ (recall $\{K\}_{C \mu^{2}}$ denotes the point in $E_{1}$ determined by the line $C \mu^{2}+K \mu t$, with $K$ a degree- $(d-1)$ curve such that $\left.K_{\mu} \nsucceq C_{\mu}\right)$. We can assume $\mu$ is the line $x_{0}=0$; also, choose forms $P$ and $Q$ of degree $d$ in $x_{0}, x_{1}, x_{2}$ representing $C \mu^{2}$ and $K \mu$. If $R_{d}$ is a form of degree $d$ in $x_{1}, x_{2}$, look at the curve $\gamma: \gamma(t)=P+Q t+R_{d} t^{2}$. Any such curve determines a point in the fiber of $E_{2}$ over $\{K\}_{C \mu^{2}}$; this identifies the $(d+1)$-dimensional vector space of forms over $\mu$ with the complement of $\widetilde{E}_{1}$ in the fiber of $E_{2}$ over $\{K\}_{C \mu^{2}}$ (the identification depends on the choice of $P$ and $Q$ ). We have seen in $\S 2$ (equation $(*)$ ) that $\gamma$ has contact of order at least 3 with the line-condition corresponding to a (general) line $l$ intersecting $\mu$ at $\left(0: l_{1}: l_{2}\right)$ if and only if (notation as in $\left.\S 2\right)$

$$
Q_{d-1}\left(l_{1}, l_{2}\right)^{2}-4 R_{d}\left(l_{1}, l_{2}\right) P_{d-2}\left(l_{1}, l_{2}\right)=0 .
$$

Let $\widetilde{L}_{l}$ be the line-condition in $V_{2}$ corresponding to $l$. In terms of the above identification, (**) gives the equation of the fiber of $\widetilde{L}_{l} \cap E_{2}$ over $\{K\}_{C \mu^{2}}$ (given $P, Q$ and $l_{1}, l_{2}$ there is an affine hyperplane of $R_{d}$ 's satisfying $(* *)$ ). Supposing that $(* *)$ is true for all $l_{1}, l_{2}$ gives the equation

$$
Q_{d-1}^{2}-4 R_{d} P_{d-2}=0
$$

for the scheme-theoretic intersection of the line-conditions in $\widetilde{V}$ over $\{K\}_{C \mu^{2}}$. As $\{K\}_{C \mu^{2}}$ moves in $B_{1}^{\circ}$, this defines a scheme $\mathscr{B}_{2}^{\circ}$ supported on $B_{2}^{\circ}$.

Claim 2. In a neighborhood of the bad points, $\mathscr{B}_{2}^{\circ}$ is the part of $\mathscr{S}_{2}$ supported on $B_{2}^{\circ}$.

Proof. It is enough to observe that $\mathscr{S}_{2}$ is the intersection $\bigcap_{l} \widetilde{L}_{l}$ of all lineconditions in $\widetilde{V}$, while by definition $\mathscr{B}_{2}^{\circ}$ is the intersection of $\mathscr{S}_{2}$ with $E_{2}$ near bad points . As seen above, $\mathscr{S}_{2} \subset E_{2}$ near such points, and the assertion follows. 
In fact, this argument shows $\mathscr{B}_{2}^{\circ}$ coincides with $\widetilde{L}_{1} \cap \cdots \cap \widetilde{L}_{2 d}$ in a neighborhood of the bad points, where $\widetilde{L}_{1}, \ldots, \widetilde{L}_{2 d}$ are general line-conditions in $\widetilde{V}_{2}$.

Now let $\mathscr{B}_{2}$ be the closure of $\mathscr{B}_{2}^{\circ}$. So far, the discussion above shows that $\widetilde{P}_{1} \cap \cdots \cap \widetilde{P}_{N-2 d} \cap \widetilde{L}_{1} \cap \cdots \cap \widetilde{L}_{2 d}$ consists of the good points and of $\widetilde{P}_{1} \cap \cdots \cap$ $\widetilde{P}_{N-2 d} \cap \mathscr{B}_{2} ;$ therefore

$$
\widetilde{P}^{N-2 d} \cdot \widetilde{L}^{2 d}=N_{d}(2 d)+\widetilde{P}_{1} \cdots \cdot \widetilde{P}_{N-2 d} \cdot\left[\mathscr{B}_{2}\right] .
$$

For the next step in our program we need to show

Claim 3. $\left[\mathscr{B}_{2}\right]=2^{d-4}\left[B_{2}\right]$.

Proof. $\mathscr{B}_{2}$ is a subscheme of $E_{2}$ of codimension $2 d-1$. We are going to cut $\mathscr{B}_{2}$ with a $(2 d-1)$-dimensional variety $Z$ intersecting the support $B_{2}$ of $\mathscr{B}_{2}$ transversally at a point $p$. To prove the assertion we must show that $Z$ intersects $\mathscr{B}_{2}$ with multiplicity $2^{d-4}$ at that point.

To obtain $Z$, we fix $\mu$ to be the line $x_{0}=0$; fix a 2-dimensional net of degree- $(d-2)$ curves $C$ cutting $\mu$ into divisors,

$$
\left(x_{1}^{2}+\alpha x_{1} x_{2}+\beta x_{2}^{2}\right)\left(x_{1}^{d-4}+x_{1}^{d-5} x_{2}+\cdots+x_{2}^{d-4}\right) ;
$$

this gives a 2-dimensional family of curves $C \mu^{2}$ in $B$, parametrized by $\alpha, \beta$, hence determining a $(d-1)$-dimensional subvariety of $B_{1}$ : all $\{K\}_{C \mu^{2}}$ with $C$ and $\mu$ as above. We define the $(d-2)$-dimensional subvariety of $B_{1}$ obtained by considering $\{K\}_{C \mu^{2}}$ with $C \mu^{2}$ as above and $K$ in the form

$$
x_{1}\left(x_{1}^{d-4}+c_{1} x^{d-5} x_{2}+\cdots+c_{d-4} x_{2}^{d-4}\right) x_{2}^{2} .
$$

This in turn determines a $(2 d-1)$-dimensional subvariety $Z$ of $E_{2}$. The reader will easily verify that $Z$ intersects $B_{2}$ transversally at a point $p$ over the point $\{K\}_{C \mu^{2}}$ determined by $\alpha=\beta=0, c_{1}=\cdots=c_{d-4}=1$.

We can parametrize the fiber over such $\{K\}_{C \mu^{2}}$ near $p$ as above by forms

$$
\frac{1}{4}\left(a_{0} x_{1}^{d}+a_{1} x_{1}^{d-1} x_{2}+\cdots+a_{d} x_{2}^{d}\right) ;
$$

this parametrizes $Z$ at $p$ by the data

$$
\left(\alpha, \beta ; c_{1}, \ldots, c_{d-4} ; a_{0}, \ldots, a_{3} ; a_{4}, \ldots, a_{d}\right) ;
$$

in these terms $p$ has coordinates $(0,0 ; 1, \ldots, 1 ; 0, \ldots, 0 ; 1, \ldots, 1)$. We can now restrict the equations for $\mathscr{B}_{2}$ to $Z$ : we get

$$
\begin{aligned}
x_{1}^{2}\left(x_{1}^{d-4}+c_{1} x^{d-5} x_{2}+\cdots+c_{d-4} x_{2}^{d-4}\right)^{2} x_{2}^{4}=\left(x_{1}^{2}+\alpha x_{1} x_{2}+\beta x_{2}^{2}\right) \\
\cdot\left(x_{1}^{d-4}+x_{1}^{d-5} x_{2}+\cdots+x_{2}^{d-4}\right)\left(a_{0} x_{1}^{d}+a_{1} x_{1}^{d-1} x_{2}+\cdots+a_{d} x_{2}^{d}\right),
\end{aligned}
$$

i.e., $2 d-1$ equations in $\alpha, \beta, c_{1}, \ldots, c_{d-4}$ and $a_{0}, \ldots, a_{d-4}$. Checking that the multiplicity of intersection of the corresponding loci at $p$ is $2^{d-4}$ is a standard computation, which we also leave to the reader.

It follows from the above that the contribution to

$$
\widetilde{P}_{1} \cap \cdots \cap \widetilde{P}_{N-2 d} \cap \widetilde{L}_{1} \cap \cdots \cap \widetilde{L}_{2 d}
$$

due to the 'bad' points ( $c$ in the statement of Lemma III(2)) is

$$
2^{d-4} \widetilde{P}_{1} \cdots \cdot \widetilde{P}_{N-2 d} \cdot\left[B_{2}\right]=2^{d-4} \widetilde{P}^{N-2 d} \cdot\left[B_{2}\right] .
$$


The computation in $N_{d}(2 d)$ will now be complete if we show

Claim. $\int_{\widetilde{V}} \widetilde{P}^{N-2 d} \cdot\left[B_{2}\right]=d(d-1)(d-3)(d-2)(d+2)$.

Proof. Observe that the general line-condition $\widetilde{P}$ in $\widetilde{V}$ is actually the pull-back of the general line-condition $P$ in $\mathbb{P}^{N}$ (indeed, the centers of the blow-ups cut point-conditions properly). Also, recall that $B_{2}$ maps down on $\mathbb{P}^{N}$ to the subvariety $T$ of $B$ consisting of $C \mu^{2}$ with $C$ tangent to $\mu$. Then, by the projection formula,

$$
\int_{\widetilde{V}} \widetilde{P}^{N-2 d} \cdot\left[B_{2}\right]=\int_{\mathbb{P}^{N}} P^{N-2 d} \cdot[T]
$$

On the other hand, consider the subvariety $T_{0}$ of $\mathbb{P}^{(d-2)(d+1) / 2} \times \check{\mathbb{P}}^{2}$ given by all pairs $(C, \mu)$ with $C$ tangent to $\mu$. Then $i_{*}\left[T_{0}\right]=[T]$ (notation as in $\S 3.1$ ), and since $i^{*} P=l+2 m$ we get, again by the projection formula,

$$
\int_{\widetilde{V}} \widetilde{P}^{N-2 d} \cdot\left[B_{2}\right]=\int_{\mathbb{P}^{(d-2)(d+1) / 2} \times \breve{\mathbb{P}}^{2}}(l+2 m)^{N-2 d}\left[T_{0}\right] .
$$

Now $\left[T_{0}\right]=(2 d-6) l+(d-2)(d-3) m$ (indeed, $2(d-2)-2$ curves of degree $d-2$ in a pencil are tangent to a given line, and $(d-2)(d-3)$ lines in a pencil are tangent to a given curve of degree $d-2)$; therefore

$$
\begin{aligned}
\int_{\widetilde{V}} \widetilde{P}^{N-2 d} \cdot\left[B_{2}\right] & =\int(l+2 m)^{N-2 d}((2 d-6) l+(d-2)(d-3) m) \\
& =d(d-1)(d-3)(d-2)(d+2) .
\end{aligned}
$$

Thus, we have shown

$$
\widetilde{P}^{N-2 d} \cdot \widetilde{L}^{2 d}=N_{d}(2 d)+2^{d-4} d(d-1)(d-3)(d-2)(d+2) .
$$

Comparing with

$$
\widetilde{P}^{N-2 d} \cdot \widetilde{L}^{2 d}=(2 d-2)^{2 d}-2^{d-3} d(d-1)\left(4 d^{4}-11 d^{3}+11 d^{2}-8 d+10\right)
$$

(from $\S 4.2$ ) gives

$$
N_{d}(2 d)=(2 d-2)^{2 d}-2^{d-4} d(d-1)\left(8 d^{4}-21 d^{3}+19 d^{2}-20 d+32\right),
$$

which concludes the proof of Theorem IV.

$$
\text { 5. } N_{4}(9)
$$

In his Almindelige Egenskaber ved Systemer af plane Kurver, Zeuthen provides an exhaustive analysis of families of plane quartics, and lists many enumerative results (several of these appear also in $[S, \S 26])$. We are very far from recovering all his results; however, for smooth quartics, Proposition 1.2 and Theorem IV in $\S 4$ give for $d=4$

$$
N_{4}(k)=6^{k}, \quad 0 \leq k \leq 6, \quad N_{4}(7)=279,600, \quad N_{4}(8)=1,668,096,
$$

in agreement with Zeuthen. In this section we indicate how to extend the construction of $\S 3$ to obtain the next characteristic number: 
Theorem V. The number of smooth quartics containing five general points and tangent to nine general lines in the plane is

$$
N_{4}(9)=9,840,040 \text {. }
$$

The result again agrees with Zeuthen's computations. To our knowledge, the remaining five characteristic numbers for smooth plane quartics still await a modern verification.

The approach is roughly the following: by the considerations in $\S 4.3, \mathscr{B}_{2}$ is generically reduced for $d=4$; it will then be easy to blow-up $V_{2}$ along $\mathscr{B}_{2}$, thereby extending the construction of a variety of complete quartics over the set of curves consisting of a smooth conic and a double line tangent to it. In the new variety, the intersection of five general point-conditions and nine general lineconditions will consist of isolated points: $N_{4}(9)$ 'good' points, the double conic containing the five points, and finitely many points corresponding to quartics consisting of a triple of distinct lines, one of which is double, meeting at a point. The contribution of the degenerate points can be computed directly, giving the result.

Executing this plan involves the same techniques we employed in the rest of the note: we will indicate the main points here, leaving many details to the reader.

5.1. The third blow-up. We keep the following notation for the rest of the note: for $d=4, S \subset \mathbb{P}^{14}$ denotes the the locus of nonreduced quartics, $B$ is the set of curves $C \mu^{2}$ containing a double line $\mu$, and $T \subset B$ is the set of curves $C \mu^{2}$ with $\mu$ tangent to $C . B^{\circ}$ is the set of quartics $C \mu^{2}$ consisting of a double line $\mu$ and of a reduced conic $C$ not containing $\mu$, and $T^{\circ}=T \cap B^{\circ}$; both $B^{\circ}$ and $T^{\circ}$ are nonsingular. Also, we will denote by $U$ the subset of $T$ consisting of quartics $C \mu^{2}$ with $C$ a singular conic, and $\mu$ a line intersecting $C$ at a singular point; and we will let $U^{\circ}=U \cap T^{\circ}$ : so points of $U^{\circ}$ are triples of distinct lines, one of which double, meeting at a point.

In Lemma II we computed the thickness of $\mathscr{S}$ at points of $B-T$ and $T-U$; the additional information we need now is

Lemma II. (4) If $p \in U^{\circ}$, then $\operatorname{th}_{p}(\mathscr{S})=4$.

The verification is left to the reader: it is analogous to the proof of Lemma II in $\S 2$.

The analogue of Lemma 1.3 in the new situation is:

Remark. The intersection of five general point-conditions and $S$ in $\mathbb{P}^{14}$ consists of an isolated point corresponding to the double conic containing the five given points, and of a 2-dimensional subset of $B^{\circ}$. This subset contains a 1-dimensional subset of $T^{\circ}$ and finitely many points of $U^{\circ}$.

In $\S 3$ we analyzed the two blow-ups over $B^{\circ}$ : by this remark that discussion takes care of the new situation as well. We get an extension of Lemma III for $d=4$ :

Lemma III. (3) Denote by $P_{2}$ and $L_{2}$ the classes of the general point-and lineconditions in $V_{2}$, resp. Then $P_{2}^{5} \cdot L_{2}^{9}$ is the sum of $N_{4}(9)$ and of a contribution due to a 1-dimensional subset of $B_{2}^{\circ}$ and to an isolated point (corresponding to a double conic). 
Recall that $B_{2}^{\circ}$ is isomorphic to $T^{\circ}$; also, $T^{\circ} \cong B_{2}^{\circ}$ is smooth for $d=4$. We are going to blow-up $V_{2}$ along $B_{2}$, and examine the preimage of points of $B_{2}^{\circ}$ : denoting by $U_{2}^{\circ}$ the subset of $B_{2}^{\circ}$ identified with $U^{\circ}, U_{2}^{\circ}$ will be the set that 'survives' the third blow-up.

Let then $V_{3} \stackrel{\pi_{3}}{\rightarrow} V_{2}$ be the blow-up of $V_{2}$ along $B_{2}$ and $\widetilde{E}_{2}$ be the proper transform of $E_{2}$ in $V_{3}$.

Proposition 5.1. Denote by $\mathscr{S}_{3}$ the intersection of all line-conditions in $V_{3}$. Then $\mathscr{S}_{3} \cap \pi_{3}^{-1}\left(B_{2}^{\circ}\right)$ is supported on a variety $B_{3}^{\circ}$ mapping bijectively onto $U_{2}^{\circ}$.

Proof. This follows from the discussion in $\S 4$.3. First observe that $\mathscr{B}_{2}^{\circ}:=$ $\mathscr{S}_{2} \cap \pi_{2}^{-1}\left(B_{1}^{\circ}\right)$ coincides with $B_{2}^{\circ}$ for $d=4$ (cf. $\S 4.3$ : we gave equations for $\mathscr{B}_{2}^{\circ}$ for all $d$; for $d=4$ the equations define a reduced scheme). As a consequence, much as in Lemma 3.2, $\mathscr{S}_{3} \cap \pi_{3}^{-1}\left(B_{2}^{\circ}\right)$ must be disjoint from $\widetilde{E}_{2}$, and therefore it consists of at most one point over each point of $B_{2}^{\circ}$. On the other hand, by Claim 2 in $\S 4.3, B_{2}^{\circ}=\mathscr{B}_{2}^{\circ}$ coincides with the scheme-intersection $\mathscr{S}_{2}$ of the line-conditions along $B_{2}^{\circ}-U_{2}^{\circ}$ : therefore $\mathscr{S}_{3} \cap \pi_{3}^{-1}\left(B_{2}^{\circ}-U_{2}^{\circ}\right)=\varnothing$. The assertion amounts then to showing that $\mathscr{S}_{3} \cap \pi_{3}^{-1}\left(U_{2}^{\circ}\right) \neq \varnothing$ : which follows from the fact that $\operatorname{th}_{p}(\mathscr{S}) \geq 4$ if $p \in U^{\circ}$ (Lemma II(4) above), similarly to Lemma 3.3.

We let $B_{3}$ be the closure of $B_{3}^{\circ}$ in $V_{3}$. From Proposition 5.1 we get the main tool for the computation:

Lemma 5.2. Let $P_{3}$ and $L_{3}$ be the classes of the general point- and line-conditions in $V_{3}$, resp. Then $P_{3}^{5} \cdot L_{3}^{9}$ is the sum of $N_{4}(9)$ and of a contribution due to finitely many points of $B_{3}^{\circ}$ and to an isolated point (corresponding to a double conic).

This follows from Lemma I, the remark at the beginning of this section, and Proposition 5.1.

5.2. $\quad P_{3}^{5} \cdot L_{3}^{9}$. The computation of $N_{4}(9)$ is now reduced to applying formula (*) in $\S 4$ (in order to compute $P_{3}^{5} \cdot L_{3}^{9}$ ), and evaluating the contribution due to the 'bad' points in $V_{3}$. The only new element needed to apply $(*)$ is an explicit realization of $T^{\circ} \cong T_{1}^{\circ} \cong B_{2}^{\circ}$.

The first two stages of the computation follow the steps of $\S \S 4.1,4.2$ :

$$
\begin{aligned}
P_{1}^{5} \cdot L_{1}^{9} & =6^{9}-\int(l+2 m)^{5} \frac{(1+6 l+12 m)^{9}(1+l)^{6}(1+m)^{3}}{(1+l+2 m)^{15}} \\
& =10,077,696-67,131=10,010,565,
\end{aligned}
$$

and (using $p_{*} e=-1, p_{*} e^{2}=-4 l-7 m$, and $p_{*} e^{3}=-10 l^{2}-38 l m-28 m^{2}$ obtained as in Lemma 4.1)

$$
\begin{aligned}
P_{2}^{5} \cdot L_{2}^{9} & =P_{1}^{5} \cdot L_{1}^{9}-\int(l+2 m)^{5} \frac{(1+6 l+12 m-e)^{9}(1+l+m-e)^{10}}{(1+e)(1+l+2 m-e)^{15}} \\
& =10,010,565-149,465=9,861,100 .
\end{aligned}
$$

To apply formula $(*)$ from $\S 4$ to the third blow-up, we need to gather information about $B_{2}^{\circ}$ : specifically, we need a compactification $\bar{T}$ of $T^{\circ} \cong T_{1}^{\circ} \cong B_{2}^{\circ}$ with a manageable Chow ring and a class restricting to $c\left(N_{B_{2}^{\circ}} V_{2}\right)$ on $T^{\circ}$.

Now, $T^{\circ}$ parametrizes pairs $(C, \mu)$, where $C$ is a reduced conic and $\mu \not \subset C$ is a line tangent to $C$. We choose for $\bar{T}$ the closure of the subset of $\mathbb{P}^{5} \times \check{\mathbb{P}}^{2} \times \mathbb{P}^{2}$ 
consisting of triples $(C, \mu, p)$, where $\mu$ is a line, $p \in \mu$, and $C$ is a smooth conic tangent to $\mu$ at $p . \bar{T}$ is smooth (as a $\mathbb{P}^{3}$-bundle over a $\mathbb{P}^{1}$-bundle over $\left.\mathbb{P}^{2}\right)$ and contains $T^{\circ}$ as an open set. The Chow ring of $\bar{T}$ is generated by the hyperplane classes $l$ of $\mathbb{P}^{5}, m$ of $\check{\mathbb{P}}^{2}$, and $k$ of $\mathbb{P}^{2}$; the reader will easily check the relations:

$$
\begin{gathered}
\int l^{4} m^{2}=2, \quad \int l^{5} m=2, \quad \int k l^{3} m^{2}=1, \quad \int k l^{4} m=3, \\
\int k l^{5}=2, \quad \int k^{2} l^{3} m=1, \quad \int k{ }^{2} l^{4}=1
\end{gathered}
$$

(all other degree-6 monomials give 0 ).

The class of the point-conditions pulls-back to $l+2 m$ via the natural map $\bar{T} \rightarrow \mathbb{P}^{5} \times \check{\mathbb{P}}^{2} \rightarrow \mathbb{P}^{14}$. We also need the pull-back (to $B_{2}^{\circ}$ ) of the classes of $E_{1}$ and $E_{2}$; the key observation is

Claim. The pull-back of the classes of $E_{1}$ and $E_{2}$ to $B_{2}^{\circ}$ coincide.

Proof. This follows from Lemma 3.2, which showed that $B_{2}^{\circ}$ is disjoint from $\widetilde{E}_{1} . \quad B_{2}^{\circ}$ is a section of $E_{2}$ over $T_{1}^{\circ}$, say $B_{2}^{\circ}=\mathbb{P}(\mathscr{L}) \subset \mathbb{P}\left(N_{B_{1}^{\circ}} V_{1}\right)=E_{2}$ for $\mathscr{L}$ a rank-1 subbundle of $N_{B_{1}^{\circ}} V_{1}$ (notation as in $\S 3.2$ ). Tautologically $\mathscr{L}=\mathscr{O}_{\mathbb{P}(\mathscr{L})}(-1)$ is the restriction of $\mathscr{O}_{\mathbb{P}\left(N_{B_{1}^{\circ}} V_{1}\right)}(-1)$, so that $c_{1}(\mathscr{L})$ is the pullback of the class of $E_{2}$; on the other hand, since $B_{2}^{\circ}$ is disjoint from $\widetilde{E}_{1}$, then $\mathscr{L}$ is transversal to $\left.N_{B_{1}^{\circ}} E_{1}\right|_{T_{1}^{\circ}}$ in $\left.N_{B_{1}^{\circ}} V_{1}\right|_{T_{1}^{\circ}}$, so $\left.\mathscr{L} \cong N_{E_{1}} V_{1}\right|_{T_{1}^{\circ}}$ : therefore $c_{1}(\mathscr{L})$ is also the restriction of the class of $E_{1}$.

By consistency with the notation of $\S 4$, we denote by $e$ a class of $\bar{T}$ restricting to the pull-back of the class of $E_{1}$ (or $E_{2}$ ) on $B_{2}^{\circ}$. At this stage we can apply $(*)$ from $\S 4$ and write

$$
P_{3}^{5} \cdot L_{3}^{9}=P_{2}^{5} \cdot L_{2}^{9}-\int_{\bar{T}}(l+2 m)^{5} \frac{(1+6 l+12 m-2 e)^{9}}{c\left(N_{B_{2}} V_{2}\right)},
$$

where $c\left(N_{B_{2}} V_{2}\right)$ is any class of $\bar{T}$ restricting to $c\left(N_{B_{2}^{\circ}} V_{2}\right)$ on $B_{2}^{\circ}$. To obtain $c\left(N_{B_{2}} V_{2}\right)$ we apply a few Euler sequences as usual. With some abuse of notation, we get:

$$
\begin{aligned}
c\left(N_{B_{2}} V_{2}\right) & =c\left(N_{T_{1}} B_{1}\right) c\left(N_{B_{1}} V_{1} \otimes \check{\mathscr{L}}\right) c(\mathscr{L}) \\
& =c\left(N_{T} B\right) c(\mathscr{G} \otimes \check{\mathscr{L}}) c\left(N_{B_{1}} V_{1} \otimes \check{\mathscr{L}}\right) c(\mathscr{L}),
\end{aligned}
$$

with $\mathscr{L}$ as in the proof of the claim and $\mathscr{G}$ as in $\S 4.2$. This gives in particular

$$
\begin{aligned}
c_{1}\left(N_{B_{2}} V_{2}\right) & =(2 l+2 m)+(4 l+7 m-2 e)+(5 l+20 m-10 e)+e \\
& =11 l+29 m-11 e .
\end{aligned}
$$

Therefore

$$
P_{3}^{5} \cdot L_{3}^{9}=P_{2}^{5} \cdot L_{2}^{9}-\int_{\bar{T}}(l+2 m)^{5} \frac{(1+6 l+12 m-2 e)^{9}}{(1+11 l+29 m-11 e+\cdots)} .
$$

Claim. $e=3 l+6 m-3 k$ on $\bar{T}$. 
Proof. Let $\mathscr{L}$ be as above; then $\mathscr{L}$ is the restriction of $\mathscr{O}_{E_{1}}(-1)$ to $T_{1}^{\circ} \cong T^{\circ}$, and $e$ restricts to $c_{1}(\mathscr{L})$. Notice that then $\mathscr{L}$ is a subbundle of (the restriction of) $\mathscr{G}$ on $T^{\circ}$, and $T_{1}^{\circ}=\mathbb{P}(\mathscr{L}) \subset \mathbb{P}(\mathscr{G})$. Now there is a natural map

$$
\bar{T} \longrightarrow \bar{T} \times \mathbb{P}^{9}
$$

sending the triple $(C, \mu, p)$ to $(C, \mu, p, C \mu)$ (thinking of $C \mu$ as a plane cubic $\left.\in \mathbb{P}^{9}\right)$. Tracing the definitions of $\mathscr{L}$ and $\mathscr{G}$, we find that $c_{1}(\mathscr{G} / \mathscr{L})$ is the pull-back to $\bar{T}$ of the divisor in $\bar{T} \times \mathbb{P}^{9}$ consisting of quadruples $(C, \mu, p, K)$, with $K$ a cubic containing $p$. Therefore

$$
c_{1}(\mathscr{G} / \mathscr{L})=l+m+3 k,
$$

and the claim follows from this and the previously known $c_{1}(\mathscr{G})=4 l+7 m$ (cf. Lemma 4.1).

We can finally deduce

$$
\begin{aligned}
P_{3}^{5} \cdot L_{3}^{9} & =P_{2}^{5} \cdot L_{2}^{9}-\int_{\bar{T}}(l+2 m)^{5} \frac{(1+6 k)^{9}}{(1-22 l-37 m+33 k+\cdots)} \\
& =9,861,100-4,526=9,856,574 .
\end{aligned}
$$

5.3. $\quad N_{4}(9)=9,840,040$. Combining the computation of $\S 5.2$ and Lemma 5.2 in $\S 5.1$, we can claim now that $N_{4}(9)=9,856,574$ minus a contribution due to finitely many points of $B_{3}$ and to a point corresponding to a double conic.

The computation of the contribution due to the points in $B_{3}$ is similar to the computation in $\S 4.3$. By Proposition 5.1, the scheme-intersection $\mathscr{H}_{3}$ of all line-conditions is supported on $B_{3}$ in a neighborhood of the points; the reader will verify that $\mathscr{S}_{3}$ is reduced in a neighborhood of these points (similarly to $\S 4.3$, one can use $(* *)$ from $\S 2$ to write equations for $\mathscr{S}_{3}$ in a neighborhood of the points), so that the contribution equals $\int_{V_{3}} P_{3}^{5} \cdot\left[B_{3}\right]$. By the projection formula, this equals $\int_{\mathbb{P} 14} P^{5} \cdot[U]$; and by the projection formula again this is

$$
\int_{\mathbb{P}^{5} \times \tilde{\mathbb{P}}^{2}}(l+2 m)^{5}\left[U_{0}\right]
$$

where $U_{0} \subset \mathbb{P}^{5} \times \check{\mathbb{P}}^{2}$ is the set of pairs $(C, \mu) \in \mathbb{P}^{5} \times \check{\mathbb{P}}^{2}$ with $\mu$ a line intersecting the (singular) conic $C$ at a singular point. The class of $U_{0}$ in $\mathbb{P}^{5} \times \check{\mathbb{P}}^{2}$ is easily found to be $3 l^{2}+3 l m$ (indeed, $l^{5}\left[U_{0}\right]=0, l^{4} m\left[U_{0}\right]=3, l^{3} m^{2}\left[U_{0}\right]=3$ ), so that the contribution equals $\int(l+2 m)^{5}\left(3 l^{2}+3 l m\right)=150$. Therefore

$$
N_{4}(9)+\text { contr. due to a double conic }=9,856,574-150=9,856,424 .
$$

Finally, we have to evaluate the contribution due to the double conic containing the five given points. Since this depends only on local data, we may compute it in $\mathbb{P}^{14}$.

Double conics form a subvariety $D \subset \mathbb{P}^{14}$, the image of the second Veronese embedding of $\mathbb{P}^{5}$. Denote by $h$ the hyperplane class in $\mathbb{P}^{5}$; then pointconditions restrict to $2 h$ on $D$. At a general point $C^{2}$ of $D$, the line-condition corresponding to a general line $\lambda$ has multiplicity 2 , and in fact its tangent cone is the union of the point-conditions corresponding to the two points of intersection of $\lambda$ and $C$. 
Now blow-up $\mathbb{P}^{14}$ along $D$. From the above it follows that the proper transforms of the line-conditions do not meet over a general point of $D$; a last application of $(*)$ from $\S 4$ then computes the contribution of a double conic to the intersection of five point-conditions and nine line-conditions by

$$
\int_{\mathbb{P}^{5}}(2 h)^{5} \frac{(2+12 h)^{9}}{(1+\cdots)}=2^{14} \text {. }
$$

Therefore

$$
N_{4}(9)=9,856,424-16,384=9,840,040,
$$

as claimed.

\section{REFERENCES}

[A] P. Aluff, The enumerative geometry of plane cubics I: Smooth cubics, Trans. Amer. Math. Soc. 317 (1990), 501-539.

[F] W. Fulton, Intersection theory, Springer-Verlag, 1984.

[KS] S. Kleiman and R. Speiser, Enumerative geometry of nonsingular plane cubics (to appear).

[S] H. C. H. Schubert, Kalkül der abzählenden Geometrie (1879), reprinted with an introduction by S. L. Kleiman, Springer-Verlag, 1979.

[Z] H. G. Zeuthen, Almindelige Egenskaber ved Systemer af plane Kurver, Kongelige Danske Videnskabernes Selskabs Skrifter-Naturvidenskabelig og Mathematisk 10 (1873), 287-393.

Department of Mathematics, The University of Chicago, Chicago, Illinois 60637

Current address: Department of Mathematics, Oklahoma State University, Stillwater, Oklahoma 74078 